\title{
Soft limbal niche maintains stem cell compartmentalization and function through YAP
}

Swarnabh Bhattacharya ${ }^{1 \#}$, Abhishek Mukherjee ${ }^{1}$, Sabrina Pisano ${ }^{2}$, Anna Altshuler ${ }^{1}$, Waseem Nasser ${ }^{1}$, Sunanda Dey ${ }^{1}$, Anna Kaganovsky ${ }^{1}$, Aya Amitai-Lange ${ }^{1}$, Michael Mimouni ${ }^{3}$, Sergiu Socea ${ }^{3}$, Peleg Hasson ${ }^{1}$, Chloe Feral $^{2}$, Haguy Wolfenson ${ }^{1 * \#}$, Ruby Shalom-Feuerstein ${ }^{1 * \#}$

*Equal contribution and * correspondence

(SB swarnabh@campus.technion.ac.il,HW haguyw@technion.ac.il, RSF shalomfe@technion.ac.il)

${ }^{1}$ Department of Genetics \& Developmental Biology, The Rappaport Faculty of Medicine \& Research Institute, Technion Integrated Cancer Center, Technion - Israel Institute of Technology, 31096 Haifa, Israel; ${ }^{2}$ Université Côte d'Azur, INSERM, CNRS, IRCAN, 06107 Nice, France; ${ }^{3}$ Department of Ophthalmology, Rambam Health Care Campus, 31096 Haifa, Israel.

\begin{abstract}
Stem cells' (SCs) decision to self-renew or differentiate largely depends on the extracellular environment and elasticity of their niche. A well-described mediator of the mechanotransduction pathway is the cotranscriptional activator Yes-associated protein (YAP), known to shuttle into the nucleus of cells grown on stiff matrices. YAP is also known to be essential for stemness, but confusingly, SCs often reside in soft niches. Furthermore, the role of matrix rigidity in niche formation and $\mathrm{SC}$ function in vivo is poorly understood. Here we report that the post-natal development of the murine corneal epithelium involves matrix stiffening and loss of YAP activity that is associated with the formation of differentiation compartment. Importantly, manipulating the matrix crosslinking enzyme, Lox, perturbed SC mark expression and resulted in loss of corneal transparency. In agreement, we found that YAP and mechanotransduction pathways are essential for stemness in the soft niche compartment, wound healing response, and dedifferentiation of committed cells into SCs following SC depletion. In vitro experiments revealed that stiffer substrates induced cytoplasmic YAP localization through activation of LATS1/2, facilitating SMAD2/3-mediated cell differentiation. Taken together, we propose that the soft environment of the corneal SC niche maintains YAP activity to support SC regulation during morphogenesis, adult homeostasis and regeneration by the niche.
\end{abstract}

\section{Highlights}

- Matrix stiffening mediates the post-natal compartmentalization of SCs and differentiated cells of the corneal epithelium 


\section{- YAP activity is indispensable for SC function, regeneration and dedifferentiation} following injury

\section{- Matrix stiffness enhances LATS1/2-mediated YAP inactivation and SMAD2/3- dependent corneal epithelial cell differentiation}

\section{- Manipulation of mechanotransduction pathways influences SC growth in vitro}

\section{Introduction}

Stem cell (SC) fate decisions are controlled by extrinsic signals derived by the local microenvironment, known as the niche (Spradling et al, 2001; Fuchs et al, 2004). When departing away from the niche, SCs sense the lack of self-renewal signals or the presence of commitment cues and respond by activating the differentiation program. Likewise, following SC loss, committed cells repopulate the niche and undergo reprogramming or dedifferentiation into bona fide SCs, and this fascinating process is mediated by niche signals (van Es et al, 2012; Rompolas et al, 2013; Nasser et al, 2018; Lin et al, 2018). The niche-specific guiding cues may be mediated by niche cell - SC interactions, unique extracellular matrix (ECM) composition, and/or the elasticity of the niche. Up to date, however, very little is known about SC - niche crosstalk and how the niche controls stemness in vivo.

In numerous cases, adult SC niches are significantly softer than their respective differentiation compartments (Eberwein et al, 2014; Bornschlögl et al, 2016; Monge et al, 2017; Gouveia et al, 2019). It is hypothesized that SCs can sense the environmental rigidity and translate this signal into a decision to self-renew or differentiate. In differentiated cells, a key response to stiff matrix sensing is the cytoplasmic (inactive) to nuclear (active) shuttling of YAP (Dupont et al, 2011; Halder et al, 2012). Moreover, YAP activity was shown to be essential in the process of SC differentiation (Brandão et al, 2019; Lorthongpanich et al, 2019). Confusingly, however, active YAP has also been linked with the undifferentiated state (Schlegelmilch et al, 2011; Zhang et al, 2011; Judson et al, 2012; Han et al, 2015), suggesting that the soft niche might regulate YAP by an alternative path. Hippo kinases (e.g. LATS1/2 kinases) can phosphorylate YAP, rendering it inactive or prone to degradation (Zhao et al, 2010). However, it is unclear if the Hippo pathway participates in mechanosensing-mediated YAP regulation, particularly in the context of the SC niche. Another critical gap of knowledge in the field is the lack of understanding of YAP downstream effectors.

These questions can be addressed using the murine cornea, which is an excellent in vivo model to study mechanobiological aspects of SCs. It has anatomically defined cellular compartments for SCs and 
differentiated cells with distinct biomechanical properties (Eberwein et al, 2014; Gouveia et al, 2019). Corneal epithelial SCs reside in the elastic limbus, at the circumferential zone peripheral to the stiffer cornea (Cotsarelis et al, 1990; Di Girolamo et al, 2015; Dorà et al, 2015; Amitai-Lange et al, 2015). In the corneal epithelial lineage, the expression pattern and function of YAP is unclear. YAP was found in the nucleus and cytoplasm of murine limbus and cornea (Kasetti et al, 2016), whereas in humans it was linked with corneal differentiation in one study (Gouveia et al, 2019) but not detected in another study (Raghunathan et al, 2014). Therefore, the role of YAP in limbal SC (LSC) differentiation, corneal homeostasis and pathology remain poorly understood.

Here we report that LSCs predominantly express nuclear YAP and it is essential for their functionality in vivo and in vitro. In contrast to other systems, high stiffness controls YAP localization through increased actomyosin contractility that activates LATS1/2 kinases, thus rendering phosphorylated inactive YAP in the cytoplasm. Loss of YAP activity facilitates cell differentiation that is mediated by activation of SMAD2/3. Hence, matrix mechanosensing influences YAP activity to regulate LSC fate in development, homeostasis and regeneration.

\section{Results}

\section{nYAP co-localizes with stemness during cornea development}

Not much is known about the limbal niche formation, while boundary formation and cell segregation play an essential role in maintaining adult tissue homeostasis (Dahmann et al, 2011; Batlle \& Wilkinson, 2012). Gene expression patterns and lineage tracing experiments implied that the limbus becomes the SC niche around post-natal day 15 (P15) and the cornea becomes mature at P60 in mice (Collinson et al, 2002; Dorà et al, 2015; Richardson et al, 2017). To better understand the process of cornea maturation, we first characterized the expression pattern of the K15-GFP transgene, which serves as a useful reporter for murine LSCs (Nasser et al, 2018a; Altshuler et al, 2021). K15-GFP transgenic animals were sacrificed, eyes were enucleated and imaged by a fluorescent binocular microscope. As expected, in the immature P15 corneas, K15-GFP was not restricted to the limbus but was detected throughout the entire limbal and central corneal epithelium (Fig. 1A, left panels). By contrast, the K15-GFP signal became sharply constrained to the limbus at P60 (Fig. 1A, right panel), suggesting that the SC niche has been fully stabilized by this time.

Previous measurements of the mature human cornea showed that the limbus was softer than the corneal center (Eberwein et al, 2014). We, therefore, suspected that a mechanical signal might be linked to the localization of the K15-GFP cells. To analyze the underlying rigidity that basal limbal/corneal layer cells may sense, the entire limbal/corneal epithelial layer was removed by EDTA treatment and the rigidity of the denuded surface was tested by atomic force microscopy. Whereas the mature P60 murine limbus 
displayed significantly lower rigidity ( $\sim \mathrm{kPa})$ compared to the corneal center $(\sim 7 \mathrm{kPa})$, the stiffness of the cornea and limbus at P15 were comparable and very low ( 1kPa) (Fig. 1B). In line with the homogenous stiffness found at P15, the mechano-responsive co-transcriptional regulator, YAP, showed similar distribution throughout the entire limbus/cornea at this stage; however, despite the low rigidity, YAP was present in the nucleus (nYAP) (Fig. 1C). This is surprising and contrasts the typical association of nYAP with stiffer matrices (Dupont et al, 2011; Elosegui-Artola et al, 2016; Totaro et al, 2017). The SC markers K15-GFP and K15 protein were detected throughout the entire limbus/cornea at P15 (Fig. 1A, C). By P60, nYAP and SC markers became restricted to the soft LSC compartment, while in the stiffer corneal center, cytosolic YAP (cYAP) was detected (Fig. 1C). To further illuminate this aspect and substantiate this observation, we investigated the expression of LATS1/2 kinases that can phosphorylate YAP (e.g. on residue S127), rendering the phosphorylated YAP (pYAP) inactive in the cytoplasm, unable to carry out its putative nuclear co-transcriptional activities (Zheng \& Pan, 2019; Ma et al, 2019). Indeed, pYAP and its active upstream kinases LATS1/2 (pLATS1/2) were predominantly found in the stiff differentiation compartment (Fig. S1A). This observation suggests that in the cornea, LATS1/2 can become activated by stiff substrates and consequently phosphorylate its known target, YAP, to prevent its nuclear translocation.

\section{Lox activity modulates compartmentalization and function of LSCs}

Lysyl oxidase (Lox) enzyme is a putative regulator of stromal rigidity. Lox catalyzes covalent crosslinking of collagen and elastin in the ECM, thereby increasing tissue stiffness (Levental et al, 2009; Baker et al, 2013; Vallet \& Ricard-Blum, 2019). To further explore whether stromal stiffening can influence tissue compartmentalization and function of LSCs, we used the irreversible pharmacological inhibitor of Lox, $\beta$ aminopropionitrile (BAPN) (Sampath Narayanan et al, 1972). BAPN was intraperitoneally injected once a day starting from P13 to P20 and K15-GFP signal was explored at the indicated times. Intriguingly, the disappearance of the SC marker K15-GFP from the corneal center was significantly attenuated (Fig. 2A). Large patches of saturated K15-GFP signal appeared at P17, while K15-GFP patches were still evident in central corneal zones by P20. Next, we aimed to substantiate these results further and explore whether matrix stiffening alone can perturb SC function and induce differentiation in the long term. To this end, we investigated the corneas of adult transgenic animals that over-express the Lox gene (Lox ${ }^{\mathrm{OE}}$ ) (Gabay Yehezkely et al, 2020). Interestingly, Lox ${ }^{\mathrm{OE}}$ corneas displayed severe opacification (Fig. 2B) that was coupled with typical signs of conjunctival cell invasion into the cornea (Fig.2C). These severe phenotypes are considered typical hallmarks of a clinical entity known as "LSC deficiency" (O'Callaghan \& Daniels, 2011; Ghareeb et al, 2020). Lox ${ }^{\mathrm{OE}}$ mice expressed cYAP in both the limbus and cornea (Fig. S2), altogether, 
suggesting that matrix stiffening plays a critical role in post-natal segregation of SC and differentiation compartments in the corneal epithelium.

\section{YAP activity is essential for corneal homeostasis and dedifferentiation.}

The loss of nYAP coupled with opacification of Lox ${ }^{\mathrm{OE}}$ corneas implied that mechano-sensing of the stiff matrix by LSCs induces their differentiation in vivo. This implies that YAP inhibition or forced activation of mechanotransduction will lead to LSC differentiation. To test this possibility, we performed subconjunctival injections (15 $\mu \mathrm{l}$ ) of either the pharmacological inhibitor of YAP (Verteporfin; $20 \mu \mathrm{M}$ ) or the Rho Activator II (Rho-Act; $1 \mu \mathrm{g} / \mathrm{ml}$ ) that is known to enhance the activation of the mechanotransduction pathway (Lessey et al, 2012; Burridge et al, 2019). Each agent (or vehicle as control) was injected once a day for 4-days. Next, eyes were enucleated and gene expression was explored by wholemount staining. As shown in Fig. 3A, markers of the outer (Cd63) and inner (K15-GFP) LSCs were drastically diminished by treatment with either Verteporfin or Rho-Act, in line with the elevated levels of the differentiation marker, K12. Moreover, both treatments resulted in decreased cell proliferation, as evidenced by lower incorporation of the nucleotide analogue 5-Ethynyl-2'-deoxyuridine (EdU) (Fig. 3A, S3A). Next, to further explore the link between YAP/mechanotransduction and LSC functionality, we tested their effect on LSCs' role in corneal regeneration. To that end, we performed large $(2 \mathrm{~mm})$ corneal epithelial debridement and followed epithelial healing by a fluorescein dye penetration test. As shown in Fig. 3B (quantification in Fig. S3B), both Verteporfin and Rho-Act significantly delayed wound healing, suggesting that $\mathrm{YAP} / \mathrm{mechanotransduction}$ pathway regulates LSC activity in vivo.

Catastrophic loss of the entire LSC population can be recovered owing to the high plasticity of corneal committed cells (Nasser et al, 2018). The latter cells repair denuded limbus, dedifferentiate into K15-GFP+ LSCs, and maintain tissue integrity only if the limbal niche is intact. However, the mechanism regulating this process by the niche is not clear. We hypothesized that upon arrival to the limbal niche, committed cells sense the limbal elastic modulus and that this signal stimulates YAP activity and reprograming of committed cells into LSCs. To explore this hypothesis, the entire limbal epithelium was surgically removed (including marginal conjunctival and bordering corneal peripheral epithelial), rendering the corneal epithelium deprived of LSCs (as shown by Nasser et al, 2018) and the expression of YAP was explored in the course of dedifferentiation process. Interestingly, the limbus was entirely repopulated by corneal committed cells that already expressed nYAP as early as 1 day post injury, and the signal became even more pronounced over time (Fig. S4), suggesting that YAP might be involved in the dedifferentiation process. To check this possibility and properly follow regeneration and the dedifferentiation processes in real-time, we used the triple transgenic K14-Cre ${ }^{\text {ERT2}}$; R26R-Brainbow ${ }^{2.1}$; K15-GFP mice (Nasser et al, 
2018). In this lineage tracing system, transient exposure of $2 / 3$-months old transgenic animals to tamoxifen (3-4 days injections) induces the stochastic and irreversible labeling of K14+ limbal/corneal basal cells with one out of four fluorescent protein-coding genes (i.e. cytoplasmic red (RFP) or yellow (YFP), membrane cyan (CFP) or nuclear green (GFP)) (Amitai-Lange et al, 2015a; Nasser et al, 2018c; Altshuler et al, 2021) (Fig. 3C, D). The centripetal renewal of the corneal epithelium by LSCs can be visualized by intravital microscopy over time. As shown in Fig. 3F (unwounded), large limbal radial clones that emerged from the K15-GFP+ limbus were evident four months post induction. To explore the involvement of YAP/mechanotransduction pathways in the dedifferentiation process, the entire limbal epithelium was removed and tissue regeneration was traced following subconjunctival injection of Verteporfin or Rho-act (or vehicle as control). Injections were performed every other day until 10 days (Fig. 3E), when recovery of K15-GFP signal peaked (see Fig. 3F and Nasser et al, 2018), and at this point, treatment was ceased to avoid potentially harmful effects of multiple eye injections. In both treatments, stripes of corneal committed cells repopulated the debrided limbal epithelium by day 1 post LSC depletion, similar to control injections (Fig. 3F, compare unwounded, wounded and day 1). Intriguingly, however, the K15-GFP recovery was almost abolished by Verteporfin and Rho-Act treatments (Fig. 3F, see days 7-15). Notably, Rho-Act treated eyes sometimes displayed more severe phenotypes, including partial corneal neovascularization (Fig. 3F, red arrows day 10), changes in stripe patterns and partial opacification (Fig. 3F, white arrows), in line with the phenotype of Lox ${ }^{\mathrm{OE}}$ mice (Fig. 2B). Moreover, on day 15 (5-days post last injection), the K15-GFP pattern was poorly revived in the limbus following Verteporfin and Rho-act treatments. This data suggests that mechanosensing of the soft limbus stimulates dedifferentiation. In contrast, inhibition of YAP activity or activation of Rho perturbed the normal mechanosensing and prevented reprogramming of corneal committed cells into LSCs.

\section{YAP is essential for the maintenance of undifferentiated LSC state}

The effects of injected pharmacology on LSC differentiation could involve cell-autonomous (effect on LSCs themselves) or non-cell autonomous mechanisms (e.g., a secondary effect mediated by niche cells). To corroborate the data and delineate YAP's underlying regulatory mechanisms and relevance to humans, we explored hereafter primary human LSC cultures (Figs. 4-7). To characterize YAP's expression pattern and function, we established primary human limbal and epidermal foreskin keratinocyte cultures grown in co-culture with mitotically inactive NIH-3T3-J2 feeder cells (Shalom-Feuerstein et al, 2011; Nagosa et al, 2017; Bhattacharya et al, 2019). In both cell cultures and in agreement with in vivo data (Fig. 1C), nYAP was observed in small-sized cells expressing the stem/progenitor cell markers K15 and P63 (Fig. 4A-B, yellow arrows). In agreement, inactive YAP (cYAP or pYAP) was observed in large size K15- and P63- 
negative differentiated cells (Fig. 4A-B, white arrows). To further corroborate this data, we explored YAP expression before and after calcium-induced LSC differentiation (Bhattacharya et al, 2019). LSCs were grown in a defined medium containing $150 \mu \mathrm{M}$ (low) calcium to minimize differentiation, whereas differentiation was induced by elevating the levels of calcium to $1.2 \mathrm{mM}$ (high) for seven days. Quantitative real-time polymerase chain reaction (qPCR) analysis confirmed successful cell differentiation hallmarked by the reduction in the levels of stem/progenitor cell markers (K15, P63) and increased expression of differentiation markers (K3 and K12) (Fig. 4C). Similarly, a decrease in K15 protein expression and an increase in K12 protein expression was observed by Western blot analysis (Fig. 4D). Importantly, nYAP was predominantly detected in undifferentiated LSCs, while differentiation enhanced a switch to cYAP (Fig. 4E-F).

Next, we performed knockdown experiments using endonuclease-digested silencing RNA (known as esiRNA) against YAP (siYAP). YAP repression was efficient and resulted in a significant reduction in expression of the stem/progenitor cell markers (K15, P63) and an increase in the expression of the differentiation markers (K3, K12) (Fig. 4G-I). To further investigate the regulation of stemness by YAP, we explored the impact of YAP knockdown on LSC colony-forming efficiency. Transfected cells were seeded at clonal density and allowed to expand for 2 weeks in co-culture with NIH-3T3-J2 feeder cells. As shown in Fig. 4J-K, colony-forming efficiency was drastically affected by YAP inhibition, indicating that YAP plays a crucial role in preserving long-term LSC proliferation. Finally, to validate the relevance of these findings to humans in vivo, we performed immunohistochemistry on human tissue sections. As shown in Fig. 4L-M, nYAP was predominantly detected in the SC compartment of the limbus and epidermis marked by K15, suggesting that YAP plays a common role in epithelial SCs. Altogether, these data strongly indicate that YAP is essential for the maintenance of an undifferentiated state and long-term proliferative capacity of LSCs.

\section{Stiffness-induced actomyosin contractility enhances cell differentiation through LATS1/2 mediated YAP phosphorylation}

The association between nYAP and soft matrix during development (Fig. 1), as well as Verteporfin's and Rho activator's impact in vivo (Fig. 3), suggest that actomyosin contractility influences YAP localization and differentiation. To test this, LSCs were cultivated on polydimethylsiloxane (PDMS) gels that recapitulated the rigidity of the human limbus ( $8 \mathrm{kPa}$, referred hereafter as “soft") or cornea (20 kPa, "stiff”) (Eberwein et al, 2014). Indeed, the stiff matrix induced a decrease in stem/progenitor cell markers (K15, P63) and increased differentiation markers (K3, K12) (Fig. 5A-B, S5), as previously reported (Gouveia et $a l, 2019)$. In agreement with the in vivo data in mice (Fig. 1A), LSCs grown on the stiffer matrix for 4-days 
displayed much lower levels of nYAP, whereas the stiff substrate drastically enhanced the levels of pLATS1/2 and induced a switch to cYAP (Fig. 5C-D). Similar to the effect of YAP inhibition in vivo, Verteporfin treatment on soft gels significantly enhanced cell differentiation and reduced the colonyforming ability of LSCs (Fig. S5C-E).

Additionally, cells formed more prominent adhesions on the stiff matrix, as evidenced by vinculin staining, suggesting that LSC differentiation is linked to activation of the mechanotransduction pathways (Fig. 5C). Next, we tested whether the rigidity-dependent YAP translocation and cell differentiation require actomyosin contractility. To this end, cells were seeded on stiff matrices and actomyosin-mediated forces were inhibited by Blebbistatin $(20 \mu \mathrm{M})$ for 4-days. Indeed, the stiffness-induced transition to mainly cYAP (Fig. 5E, G) and cell differentiation (Fig. 5E, H) were significantly attenuated by Blebbistatin. Moreover, treatment with Rho-Act $(1 \mu \mathrm{g} / \mathrm{ml})$ on the soft matrix resulted in enhanced pLATS1/2, cYAP (Fig. 5F-G) and cell differentiation (Fig. 5F, I). Collectively, these data indicate that stiffness-induced differentiation was controlled by LATS1/2 mediated cYAP localization through actomyosin contractility.

We next turned to characterize the forces that the LSCs produce for mechanosensing. For that purpose, we used arrays of stiff and soft fibronectin-coated elastic PDMS pillars (Wolfenson et al, 2016; Meacci et al, 2016). After overnight incubation, the cell size was significantly smaller on the softer pillars and a clear inverse correlation between cell area and K15 expression was evident (Fig. S6A-B). Moreover, larger K15negative differentiated cells typically displayed filamentous actin (F-actin) distributed as rings around individual pillars at the cell edge; in contrast, smaller K15+ cells typically showed much fewer F-actin rings, and rarely at the edge (Fig. 5J). F-actin around pillars is positively correlated with the level of the force on the pillars (Feld et al, 2020); indeed, the cells that lacked K15 expression generated on average almost 2-fold higher forces on individual pillars compared to cells that had high K15 expression (Fig. 5K). This observation is in line with the formation of larger adhesions on the stiff matrix (Fig. 5C), which support the transmission of higher contractile forces. Altogether, these data suggest that soft matrices support the formation of small adhesions and weaker contractile forces that render low LATS1/2 activity and consequently favor stemness through maintaining nYAP.

\section{SMAD2/3 mediates Stiffness-induced differentiation}

As mentioned above, the presence of nYAP on soft matrices contrasts previous findings that linked high stiffness and high forces with YAP translocation to the nucleus; however, in those cases, the mechanotransduction pathway was not dependent on LATS1/2 (i.e., the Hippo pathway) (Dupont et al, 2011). In contrast, in the case of LSCs, the control of YAP localization through mechanosensing was linked 
to LATS1/2 phosphorylation (Fig. 5). We, therefore, suspected that another signaling pathway was overriding nYAP translocation on stiff matrices, thereby leading to a unique response of the LSCs. We focused on the TGF $\beta$ pathway since recent studies showed that inhibiting SMAD2/3 attenuated epithelial cell differentiation and that there is synergy between myosin II and TGF- $\beta$ in the regulation of SC differentiation (Mou et al, 2016; Zhang et al, 2018). Typically, active phosphorylated SMAD2/3 proteins translocate to the nucleus, where they regulate transcription (Massagué, 1998). Indeed, nuclear expression of SMAD2/3 became predominant following 4-days growth on stiffer gels or otherwise by treatment with Rho-Act on soft gels (Fig. 6A, B). In agreement, calcium-induced LSC differentiation on plastic also enhanced nuclear SMAD2/3 expression (Fig. S7A, B). Next, we investigated whether SMAD2/3 was sufficient to induce differentiation. We seeded LSCs on the soft matrix and treated them with recombinant TGF $\beta$ protein for 48 hours. As expected, TGF $\beta$ induced robust nuclear SMAD2/3 expression and cell differentiation (Fig. 6C-F), whereas pharmacological inhibition of the TGF $\beta$ pathway by SB431542 on stiff matrix prevented nuclear SMAD2/3 and inhibited differentiation (Fig. 6G-J). Moreover, inhibition the TGF $\beta$ pathway by SB431542 enhanced nYAP by inhibiting phosphorylation of LATS1/2 (Fig. 6K-N), suggesting that SMAD2/3 represses nYAP localization and induces differentiation. Vice versa, inhibition of YAP activity by verteporfin in soft substrate induced nSMAD2/3 localization (Fig. 6O, P), indicating that YAP maintains stemness by preventing nSMAD2/3 localization. Finally, immunohistochemistry demonstrated that the nuclear SMAD2/3 signal is absent in the limbus whereas it is prominent in the cornea in vivo (Fig. 6Q). Altogether these data suggest that stiff matrices induce LSCs differentiation by activating SMAD2/3 through actomyosin contractility.

\section{Inhibition of mechanosensing and TGF $\beta$ pathway attenuated LSC differentiation on plastic}

Finally, our results led us to consider the fact that plastic dishes that possess extremely high stiffness (Giga Pascals) are widely used to grow LSCs for research and cell therapy. Therefore, we examined the impact of inhibition of mechanosensing by Blebbistatin or inhibition of the TGF $\beta$ pathway by SB431542 on plastic dishes. Cells were cultivated with each inhibitor for 4-days before harvesting and analysis. Treatment with either of the inhibitors resulted in enhanced expression of LSC markers, reduced differentiation gene expression by immunostaining (Fig. 7 A, E), qPCR (Fig. 7 B, F) and augmented colony-forming capacity (Fig. 7 C, G) which was further quantified (Fig. 7 D, H). Taken together, these data indicate that LSC culture on materials that extensively differ in their mechanical properties from the native niche but that are widely used in research and cell therapy may affect self-renewal signals and induce cell differentiation. Nevertheless, we now provide novel insights into optimizing LSC cultures by manipulating mechanosensing, YAP and TGF $\beta$ pathways, thereby preventing undesired cell differentiation. 


\section{Discussion}

SCs can sense the external environment and respond by making cell fate decisions to self-renew or differentiate. A relatively diminutive movement away from the niche to the differentiation compartment (e.g. of 20-30 microns) could sufficiently induce the commitment to the differentiation of Lgr5-GFP+ gut (Barker et al, 2007), K15-GFP+ hair follicle (Morris et al, 2004), or limbal SCs (Nasser et al, 2018). The function of the niche may become even more striking following SC depletion, where committed epithelial cells e.g. gut (Tetteh et al, 2016), corneal (Nasser et al, 2018), skin (Blanpain \& Fuchs, 2014; Lin et al, 2018) underwent dedifferentiation and maintained homeostasis for long term periods. However, the nature of such niche signals is primarily unknown.

We propose that to "read" and respond to the rigidity of the underlying matrix, LSCs must continuously test the microenvironment and especially the underlying ECM by applying forces, engaging the Rho /actomyosin mechanotransduction pathways. In line with the previous report (Gouveia et al, 2019), the mechanosensing of rigid substrate by LSCs enhanced cell differentiation. However, the present study supports a model in which YAP prevents LSC differentiation. This conclusion is supported by the observation that (i) nuclear localization of YAP was mainly inspected in LSCs while cytoplasmic localization of YAP was more pronounced in the differentiated cells, (ii) YAP inhibition by siRNA or pharmacological inhibitor reduced colony formation efficiency and wound healing response of LSCs that (iii) displayed enhanced differentiation phenotype, and (iv) manipulation of Rho/ROCK/actomyosin pathway influenced nuclear/cytosol expression of YAP and cell differentiation.

Seeding of LSCs on a stiffer gel (20 kPa) reduced nuclear localization of YAP. However, LSCs that were co-cultured with NIH-3T3-J2 feeder cells on a plastic dish (Giga Pascals) expressed high levels of nuclear YAP on the colony periphery (compare Fig. 4A and Fig. 5C), suggesting that feeder cells provide an essential signal that controls YAP localization and stemness. Indeed, the induction of differentiation by calcium (on a plastic dish without feeder cells) was more forceful than differentiation induced by stiffer gel (compare Fig. 4C, D with Fig. 5A, B). Altogether, these observations align with a model where the biomechanical signals act in concert with other external signals mediated by niche-dependent cells or soluble factors.

Interestingly, the cornea's stiffness was observed to be enhanced by 8 fold during maturation, suggesting that the corneal basement membrane's stiffness might be an essential niche signal regulating stemness (Fig. 1B). In agreement with in vitro and in vivo findings in humans, nYAP was observed in the soft compartment and expressed by K15 positive cells in the murine cornea at P15 and P60, indicating that YAP might play a crucial role in murine corneal development and homeostasis (Fig. 1C). In a recent publication, YAP's importance in cornea development was evident as single-copy deletion of the Yapl gene in mice induced 
complex ocular abnormalities, including microphthalmia, thinner Descemet's membrane, and corneal fibrosis, the latter phenotype being reminiscent of LSC deficiency (Kim et al, 2019). This observation is in line with the phenotype of Lox transgenic mice (Fig. 2B, C). Inhibition of YAP by substrate stiffening or Rho-activator, or by siRNA or verteporfin, induced LSC differentiation, loss of proliferation, and impaired wound healing response. This observation was in line with the positive role of YAP in maintaining stemness in other adult tissues (Camargo et al, 2007; Schlegelmilch et al, 2011; Zhang et al, 2011; Judson et al, 2012; Han et al, 2015; Totaro et al, 2017; Walko et al, 2017). Contrastingly, in some tissues, nYAP was found in differentiated cells grown on stiff matrices (Dupont et al, 2011; Elosegui-Artola et al, 2016; Totaro et al, 2017); in those cases, nYAP was correlated with adhesion growth (Elosegui-Artola et al, 2016) and large cell areas (Dupont et al, 2011). Curiously, even though YAP was cytoplasmic in differentiated LSCs grown on the stiff matrix, they still displayed larger cell-matrix adhesions and larger cell areas than LSCs on the soft matrix (Fig. 5C, S6). This apparent discrepancy indicates that YAP plays a vital role as a mechanotransduction molecule, but the outcome of its activity depends on tissue-specific co-regulators.

Interestingly, the de novo expression of nYAP by corneal committed cells already 1-day post-injury preceded K15-GFP recovery. In line, inhibition of YAP activity or forced activation of mechanotransduction prevented proper dedifferentiation of cells. This finding suggests that the soft limbus provides a dedifferentiation signal, perhaps combined with other niche signals that initiated nYAP expression and dedifferentiation, in line with the reported role in adult hepatocytes cell plasticity (Yimlamai et al, 2014). However, the question of how YAP modulates these processes and the involvement of SMAD2/3 remains to be explored.

According to the current dogma, YAP is typically bound to TAZ and both YAP/TAZ cannot bind to the DNA by themselves, they require additional factors to exert their function on gene expression. Several transcription factors have been indicated to be YAP targets, including P73, the ErbB4 cytoplasmic domain, and TEAD (Vassilev et al, 2001; Komuro et al, 2003; Basu et al, 2003). Particularly of interest, YAP has been shown to directly bind and regulate P63 expression in keratinocytes (Tomlinson et al, 2010) and adult lung basal stem cells (Zhao et al, 2014). Moreover, YAP is also implicated in regulating SOX2 expression by binding to its promoter (Bora-Singhal et al, 2015). Interestingly, SOX2 and P63 are known to control LSC proliferation and stemness (Bhattacharya et al, 2019) and it is tempting to speculate that YAP may bind to P63/SOX2 to mediate stemness and/or that loss of nYAP allows SMAD2/3-mediated repression of LSC gene activation. Future studies will be needed to elucidate YAP's target genes in LSCs and unravel the molecular circuitry controlled by YAP in the cornea.

Our study indicated that stiffness-induced actomyosin contractility leads to cYAP and nuclear expression of SMAD2/3. Further examination revealed that cYAP was mediated by LATS1/2 activity induced by 
actomyosin contractility. This observation indicates that in undifferentiated LSCs, the Hippo pathway is inactive and activation of LATS1/2 in stiff matrix induced YAP phosphorylation and sequestered YAP in the cytosol. In a previous report, YAP translocation through mechanotransduction was shown to be independent of the Hippo pathway in mesenchymal SCs (Dupont et al, 2011). Nonetheless, in this study, mechanotransduction and hippo pathways were linked, suggesting that YAP translocation can be regulated differently depending on cell type.

How stiffness induces SMAD2/3-mediated differentiation was not explored in this study. However, the role of SMAD2/3 in differentiation was evident, as inhibition of TGF $\beta$ pathway by SB431542 prevented differentiation even on stiff matrix. Although forced activation of actomyosin contractility to the cells grown on soft matrix enhanced nSMAD2/3, inhibition of TGF $\beta$ receptor on stiff matrix prevented actomyosin contractility mediated nSMAD2/3. This suggests that TGF $\beta$ ligand-mediated phosphorylation of SMAD2/3 drives its nuclear localization and induces differentiation, as evident in Fig. 6C. TGF $\beta$ is known to bind a latency-associated peptide and the latent TGF $\beta$ binding-protein-1 (Shi et al, 2011) and $\alpha v \beta 6$ integrin dependent stiffness induced contractility has been shown to liberate TGF $\beta$ in epithelial cells (Giacomini et al, 2012). Interestingly, $\alpha \mathrm{v} \beta 6$ is also expressed by corneal epithelial cells (Stepp, 2006) and they were also found to secrete latent-TGF $\beta$-binding protein through extracellular vesicles (Han et al, 2017). Moreover, a recent study observed an increase in TGF $\beta$ expression in corneal keratocytes upon an increase in substrate stiffness (Maruri et al, 2020). A similar mechanism might be at play in the corneal epithelium for stiffness induced differentiation mediated by SMAD2/3.

This study predicts that increased rigidity of the limbal stroma will lead to unavoidable LSC differentiation. Indeed, stiffening of the limbus was found following alkali burn and can result in limbal stem cell deficiency (LSCD) (Fatima et al, 2007). Interestingly, a recent study reported on the manipulation of limbal stromal stiffness via collagenase treatment in vivo (Gouveia et al, 2019), which is expected to reduce collagen fibers crosslinking in the limbal/corneal stroma and reduce ECM rigidity, enhance stemness, and reduce symptoms of experimentally induced LSCD. Furthermore, inhibition of ROCK and TGF $\beta$ pathway have been implicated in long-term expansion of epithelial cells (Zhang et al, 2018) and corneal differentiation of human pluripotent SCs (Vattulainen et al, 2019). In line, our finding provides an important mechanistic insight into the pathways regulated by such treatments and the involvement of YAP and SMAD2/3 as a consequence of mechanotransduction and TGF $\beta$ pathway inhibition. The present study collectively suggests that manipulating mechanotransduction pathways may significantly improve SC expansion, allowing optimized therapeutic application and a better understanding of pathologies that involve SC deficiency. 


\section{Methods}

\section{Animal and tissue processing}

Animal care and use conformed to the ARVO Statement for the Use of Animals in Ophthalmic and Vision

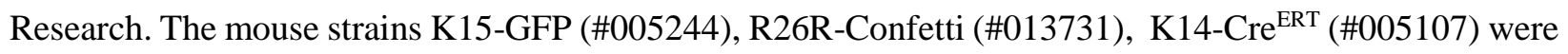
from JAX (Bar Harbor, ME). Lox ${ }^{\mathrm{OE}}$ was previously described (Gabay Yehezkely et al, 2020). Cre recombinase activity was induced by injecting intraperitoneally $(200 \mu \mathrm{l}), 4 \mathrm{mg} /$ day Tamoxifen (T5648, Sigma, St. Louis, MO) dissolved in corn oil for 3-4 consecutive days, as previously reported (Amitai-Lange et al, 2015). For wounding, mice were anesthetized (2\% Isoflurane) and injected intramuscularly with analgesic Buprenorphine $(0.03 \mathrm{mg} / \mathrm{ml}, 50 \mu \mathrm{l})$. Limbal and central corneal wounding ( $2 \mathrm{~mm}$ diameter) was performed using an ophthalmic rotating burr (Algerbrush) under fluorescent binocular. The wounded corneas were stained (1\% fluorescein) and the wounded area was imaged and quantified (NIS-Elements analysis D and ImageJ software). For BAPN experiments, intraperitoneal injection of Veh or BAPN (Sigma) $15 \mathrm{mg} / \mathrm{kg} /$ day was performed. For sub-conjunctival injection, the bulbar conjunctiva was pulled using forceps and $15 \mu$ of Rho Activator II (Cytoskeleton, CN03-B), or Verteporfin (Sigma, ML0534) was injected under the binocular using a 30-gauge needle connected to $1 \mathrm{~mL}$ syringe. Slow injection into the space between the conjunctiva and the sclera was performed to create a ballooning effect in the peri-limbal conjunctival zone.

For immunohistochemistry, paraffin sections $(5 \mu \mathrm{m})$ of mouse cornea and human corneas, skin and conjunctiva were used and proceeded as previously described (Cohen-Kaplan et al, 2008). The following primary antibodies were used mouse anti-YAP (1:200, Santa cruz biotechnology, sc-101199), mouse antiYAP (1:200, Abnova, H00010413-M01), rabbit anti-PhosphoYAP (1:1000, Cell signaling, \#13008), rabbit anti-K15 (Santa cruz biotechnology, sc-47697), rabbit anti-SMAD2/3 (1:400, Abcam, ab202445), rabbit anti-Phospho-LATS1 (1:400, Enco, 50-198-8119). Images were acquired by a Nikon ECLIPSE microscope and Digital Sight Camera (Nikon, NY, USA).

\section{Cell culture, differentiation, transfection and Real-time PCR}

Human limbal rings from cadaveric corneas were obtained post-mortem under the approval of the local ethical committee. Human tissue was handled according to the tenets of the Declaration of Helsinki. Primary human limbal and epidermal stem cells were isolated, grown and differentiated as previously described (Nagosa et al, 2017; Bhattacharya et al, 2019). For controlled calcium-induced differentiation, cells were switched to a defined medium with supplements (SCMK001, Millipore, United States) containing $1 \%$ penicillin/streptomycin and low calcium $(150 \mu \mathrm{M})$. Cells were seeded and grown to $80-100 \%$ confluency for differentiation and then switched to high $(1.2 \mathrm{mM})$ calcium for up to 1 week. Cells were 
grown on defined media and collected at indicated time points after treatment with vehicle or indicated factors Blebbistatin (para-nitroblebbistatin, Optopharma Ltd), Rho Activator II (Cytoskeleton, CN03-B), or Verteporfin (Sigma, ML0534) and taken for the Clonogenicity test (Amelio et al, 2012; Lena et al, 2008). For transfections, cells were seeded on plastic dishes and the next day transfected (Lipofectamine RNAimax, ThermoFischer) with 50nM esiRNA against EGFP (EHUEGFP, Sigma) or esiRNA against YAP (EHU113021, Sigma). Cells were collected 48-72 hrs after transfection. Real-time PCR analysis was as previously reported (Bhattacharya et al, 2019).

\section{Western blots and immunostaining}

Primary human limbal cells were washed with cold PBS and lysates were obtained and further prepared as previously reported (Bhattacharya et al. 2019). Antibodies used were rabbit anti-K15 (1:1000, Santa cruz biotechnology, sc-47697), rabbit anti-K12 (Abcam, ab185627), mouse anti-YAP (1:400, Santa cruz biotechnology, sc-101199) and rabbit anti-GAPDH (1:1000, Cell signaling, \#2118).

For in-vitro staining, cultured epithelial cells were grown on glass coverslips or Polydimethylsiloxane ( PDMS) gels and fixed in 4\% paraformaldehyde (PFA) (Sigma) for 15 minutes and then permeabilized with $0.1 \%$ Triton X-100 (BioLab) in PBS for 10 minutes. Blocking was done with bovine serum albumin (Biological Industries), 3\% donkey serum (Jackson) and 0.1\% Triton X-100 for at least 30min. Following these treatments, cells were incubated for overnight with primary antibody at 4 degrees and further incubated with secondary antibodies (1:500) for 1 hour followed by 4',6-diamidino-2-phenylindole (DAPI) or phalloidin 488 (1:400, Thermo Fisher, A12379) staining, and mounting (Thermo Scientific). Following primary antibodies were used mouse anti-YAP (1:400, Santa cruz biotechnology, sc-101199), mouse antiYAP (1:400, Abnova, H00010413-M01), rabbit anti-PhosphoYAP (1:1000, Cell signaling, \#13008), rabbit anti-Phospho-LATS1 (1:400, Enco, 50-198-8119), rabbit anti-VINCULIN (1:300, Thermo Fisher. \#700062), rabbit anti-K12 (1:400, Abcam, ab185627), rabbit anti-K15 (1:500, Santa cruz biotechnology, sc-47697), mouse anti-P63 (1:400, Santa Cru, sc-8431), rabbit anti-SMAD2/3 (1:400, Abcam, ab202445). For wholemount staining, the cornea was isolated and fixed ( $2 \%$ formaldehyde) for 2 hours, room temperature followed by permeabilized ( $0.5 \%$ Triton, 5 hours). Blocking was done for 1 hour $(0.1 \%$ TritonX-100, 2\% Normal donkey serum, 2.5\% BSA) and then incubated with primary antibody overnight, $4^{\circ} \mathrm{C}$ on a shaker and further incubated with secondary antibodies (1:500, 1 hour), followed by DAPI, tissue flattening under a dissecting binocular and mounting (Thermo Scientific), as previously described (Nasser et al, 2018). Following primary antibodies were used mouse anti-K4 (1:100, Abcam, ab183329), mouse anti-CD63 (1:100, Santa cruz biotechnology, sc-5275) and rabbit anti-K12 (1:400, Abcam, ab185627). For EdU staining, a Single intraperitoneal injection of $200 \mu \mathrm{l}(7.5 \mathrm{mg} / \mathrm{ml})$ EdU (Sigma) was performed and 6 
hours later, tissues were processed. Isolated corneas were fixed (2\% PFA, 1 hour) and stained (Click-iT, Invitrogen) according to the manufacturer's instructions followed by wholemount staining protocol (described above) for other markers staining. Images were taken by Nikon Eclipse NI-E upright microscope and Zeiss LSM880 confocal microscope. For quantification three to five different fields were imaged from different experiments and the indicated mean fluorescence intensity was calculated by ImageJ software.

\section{Tissue stiffness measurement by Atomic Force Microscopy (AFM)}

Eyes were collected from 2 and 6 weeks old mice $(\mathrm{C} 57 \mathrm{Bl} / 6 \mathrm{~J})$ following $\mathrm{C}_{2}$ sacrifice. Corneas with surrounding limbus were isolated as previously described (Amitai-Lange et al, 2015), then treated for $30 \mathrm{~min}$ in $2.5 \mathrm{mM}$ EDTA in PBS at 37C to allow epithelium removal. Next, samples were prepared by dissecting radially the tissue in two halves, each of them containing both the limbus and the cornea. The two halves were placed on SuperFrost Plus adhesion slides (Thermo Scientific) side by side and at 180 degrees to each other to have for both the areas of interest, the limbus and the cornea, free access to the sample for the AFM probe. The samples were allowed to adhere to the charged glass for 20', then the fragment of glass containing the mounted tissues was cut out from the glass slide and glued on the bottom of a $50 \mathrm{~mm}$ dish (Willco Glass Bottom Dish). Before measurements the specimen was first rinsed and after covered with $4 \mathrm{ml}$ of PBS 1x. After determining both the deflection sensitivity of the AFM system in PBS 1x using a clean glass slide fragment glued on Willco Glass Bottom Dish and the spring constant of the AFM cantilever by means of the thermal tune method, the sample was mounted on the AFM system and after thermal stabilization, for each limbus and the corresponding cornea ( $\mathrm{n}=3)$, a minimum of 3 different areas were analyzed using the "Point and Shoot" method, collecting on average 100 force-distance curves at just as many discrete points spaced by at least $20 \mu \mathrm{m}$. Force-distance curves were collected on samples using a velocity of $2 \mu \mathrm{m} / \mathrm{s}$, in relative trigger mode and by setting the trigger threshold to $1 \mathrm{nN}$. The apparent Young's modulus was calculated using the NanoScope Analysis 1.80 software (Bruker Nano Surfaces, Santa Barbara, CA, USA) applying to the force curves, after the baseline correction, the Hertz spherical indentation model using a Poisson's ratio of 0.5. All the force-distant curves having a not clear base line, a maximum above or below $1 \mathrm{nN}$ or a change of slope in the region of the fitting (minimum and maximum force fit boundary $0 \%$ and $25 \%$, respectively) were rejected and not considered for the analysis. Only the apparent Young's modulus values corresponding to a fit with $\mathrm{R}^{2}>0.85$ were considered for the analysis. The tissue mechanical proprieties were obtained by using a Bioscope Catalyst (Bruker Nano Surfaces, Santa Barbara, CA, USA), coupled with an inverted optical microscope (Leica DMI6000B, Leica Microsystems Ltd., UK). The force-distance curves needed to calculate the apparent Young's modulus were collected 
using a Borosilicate Glass spherical tip ( $5 \mu \mathrm{m}$ of diameter) mounted on a cantilever with a nominal spring constant of $0.06 \mathrm{~N} / \mathrm{m}$ (Novascan Technologies, Ames, IA USA).

\section{Polydimethylsiloxane (PDMS) substrate preparation}

To obtain PDMS substrates of different stiffness, various ratios of silicone base and crosslinking agent were used: 1:1.8 for 8 kilo Pascal $(\mathrm{kPa})$, and 1:2.5 for $20 \mathrm{kPa}$. After thorough mixing of both components, air bubbles were eliminated by application of vacuum for $30 \mathrm{~min}$. Gels were spread on glass-bottom dishes followed by incubating at $70{ }^{\circ} \mathrm{C}$ for $2 \mathrm{~h}$. Then the gels were sterilized by $30 \mathrm{~min}$ immersion in $70 \%$ ethanol. The PDMS gels' surfaces were coated with $10 \mu \mathrm{g} / \mathrm{ml}$ fibronectin for at least $1 \mathrm{~h}$ before seeding cells.

\section{Pillar arrays fabrication}

Pillar fabrication was done by pouring PDMS (Sylgard 184, Dow Corning; mixing ratio - 10:1) into silicon molds (fabricated as previously described (Ghassemi et al, 2012) with holes at fixed depths and distances. The molds were then placed, face down, onto glass-bottom $35 \mathrm{~mm}$ dishes (\#0 coverslip, Cellvis) which were incubated at $65^{\circ} \mathrm{C}$ for $12 \mathrm{~h}$ to cure the PDMS. The molds were next peeled off while immersed in ethanol to prevent pillar collapse. The ethanol was then replaced by serial dilutions with PBS, and human plasma full-length fibronectin (Merck) was added to the dish at a final concentration of $10 \mu \mathrm{g} / \mu \mathrm{l}$ for a $1 \mathrm{~h}$ incubation at $37^{\circ} \mathrm{C}$. Next, residual fibronectin was washed away by replacing the buffer to defined media with $20 \mathrm{mM}$ HEPES.

All pillars had a diameter of $2 \mu \mathrm{m}$, and heights of 5.3 or $13.2 \mu \mathrm{m}$. We used $2 \mu \mathrm{m}$ diameter pillars as these can be used to measure the long-term time-dependent forces that are generated after initial formation and reinforcement of the adhesions (Feld et al, 2020). The center-to-center spacing between pillars was $4 \mu \mathrm{m}$. Pillar bending stiffness, k, was calculated by Euler-Bernoulli beam theory:

$$
k=\frac{3}{64} \pi E \frac{D^{4}}{L^{3}}
$$

where $\mathrm{D}$ and $\mathrm{L}$ are the diameter and length of the pillar, respectively, and $\mathrm{E}$ is the Young's modulus of the material (=2 MPa for the PDMS used here).

\section{Pillar displacement measurements}


For measuring forces by K15-positive and K15-negative cells, the cells were grown in low and highcalcium media, respectively, for 4 days. On the day of the experiment, the cells were trypsinized, centrifuged with growth medium, and then resuspended and pre-incubated in defined media with HEPES at $37^{\circ} \mathrm{C}$ for $30 \mathrm{~min}$ before plating them on the $5.3 \mu \mathrm{m}$ fibronectin-coated pillars.

Time-lapse imaging of cells spreading on the pillars was performed using an inverted microscope (Leica DMIRE2) at $37^{\circ} \mathrm{C}$ using a $63 \times 1.4$ NA oil immersion objective. Brightfield images were recorded every 10 seconds with a Retiga EXi Fast 1394 CCD camera (QImaging). The microscope and camera were controlled by Micromanager software (Edelstein et al, 2010). In all cases, we imaged cells that were not in contact with neighboring cells when plated on the substrates. For each cell, a movie of 1-3 hours was recorded. To minimize photodamage to the cells, a $600 \mathrm{~nm}$ longpass filter was inserted into the illumination path.

Tracking pillar movements over time was performed with ImageJ (National Institutes of Health) using the Nanotracking plugin, as described previously (Ghassemi et al, 2012). In short, the cross-correlation between the pillar image in every frame of the movie and an image of the same pillar from the first frame of the movie was calculated, and the relative $\mathrm{x}$ - and y-position of the pillar in every frame of the movie was obtained. To consider only movements of pillar from their zero-position, we only analyzed pillars that at the start of the movie were not in contact with the cell and that during the movie the cell edge reached to them. Drift correction was performed using data from pillars far from any cell in each movie. For each pillar, the displacement curve was generated by Matlab (MathWorks).

\section{Statistical analysis}

All experiments were performed at least three times. All quantifications represent the mean \pm standard error of the mean (s.e.m.) or as indicated in the legends. Images are representative of experiments that have been repeated at least three times. Data were tested for normality where applicable by the Shapiro-Wilk method. Group comparison was performed using a two-tailed unpaired Student's $t$ test or Mann Whitney test as indicated in the legends. Multiple groups comparison was performed by ANOVA followed by Tukey's test. Differences were considered to be statistically significant from a $p$-value below 0.05 .

\section{Acknowledgment}

We thank D. Aberdam for the critical reading of the manuscript. RSF has received funding from the Israel Science Foundation (1308/19 and 2830/20), Rappaport Family Institute for Research in Medical Sciences, NIH-exploratory R21 (800040), European Union's Horizon 2020 research \& innovation programme 
(828931). HW acknowledges support from the Israel Science Foundation (1738/17) and from the Rappaport Family Institute for Research in Medical Sciences; HW is an incumbent of the David and Inez Myers Career Advancement Chair in Life Sciences. CF was supported by INCA (Institut national du cancer) PL-Bio \#2019-11/368/NI-HO, and the French Government (National Research Agency, ANR) through the "Investments for the Future" programs LABEX SIGNALIFE ANR-11-LABX-0028-01 and IDEX UCAJedi ANR-15-IDEX-01. The atomic force microscopy of IRCAN was supported by the Association pour la Recherche sur le Cancer (ARC), by the Infrastructures en Biologie Santé et Agronomie (IBiSA) and by the Conseil Départemental 06 de la Région Provence Alpes-Côte d'Azur. PH has received funding from the Israeli Science Foundation grant number 1111/18.

Author Contribution: SB was involved in conceptual and experimental design, performed and interpreted majority of the experiments, prepared the figures, analyzed data, and participated in the manuscript writing; AM performed the pillar experiments, analyzed data, and participated in ongoing discussions about the study; SP, CF performed AFM experiment, analyzed data, prepared the figures, and participated in the discussion and manuscript writing; AA, WN, SD, AAL performed experiments and provided data; MM, SS performed subconjunctival injections; AK, PH provided materials and participated in discussions and manuscript writings; HW and RSF were involved in conceptual and experimental design, data interpretation, and participated in manuscript writing. All authors approved the manuscript. 


\section{Figure Legends}

Figure 1. The Development of SC and differentiation compartment is linked with a switch in rigidity and YAP localization. (A) K15-GFP expression pattern in the murine cornea at P15 and P60. Green and white arrowheads indicate limbus and cornea region respectively. (B) Comparison between the limbus and the cornea mechanical properties. Mean $+/-$ SD of the apparent Elastic modulus (in $\mathrm{kPa}$ ) of the limbus and cornea of P15 on the left, and P60, on the right (the higher the Eapp the stiffer the tissue). The plot shows the merge of three experiments performed on as many independent samples, using the same AFM setup (deflection sensibility and cantilever spring constant). (C, D) Immunohistochemistry of K15 and YAP protein was performed on paraffin sections of the P15 or P60 mouse cornea. Golden arrowheads indicate nuclear YAP and cytoplasmic K15 staining in the basal cells of the limbus and cornea at P15 and at the

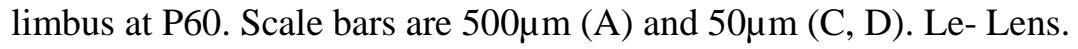

Figure 2. Modulation of Lox crosslinking activity disrupts SC compartmentalization and function. (A) K15-GFP expression pattern in murine cornea treated with vehicle or BAPN from P13 to P17 (A) or P20 (B). Green and white arrowheads indicate limbus and cornea region respectively. Brightfield image of $\mathrm{WT}$ and $\mathrm{Lox}^{\mathrm{OE}}$ cornea displaying conjunctivalization. White arrowhead indicates invading conjunctiva and opacification. (E) Wholemount immunostaining for markers of the conjunctiva (K4) and cornea (K12) was performed in WT and Lox ${ }^{\mathrm{OE}}$ mice. Scale bars are $500 \mu \mathrm{m}(\mathrm{A})$ and $50 \mu \mathrm{m}(\mathrm{B}, \mathrm{C})$. Le- Lens.

Figure 3. Interference of mechano-transduction pathway through YAP inhibitor or Rho activator impaired LSC function and dedifferentiation. (A) Sub-conjunctival injections of $20 \mu 1$ of each verteporfin $(20 \mu \mathrm{M})$ or Rho-Act $(1 \mu \mathrm{g} / \mathrm{ml})$ (or vehicle as control) was injected once a day for 4-days on K15GFP transgenic mice. Next, wholemount immunostaining was performed for quiescent stem cell marker (CD63) and differentiation marker K12 or EdU to access proliferation on the whole cornea. EdU was injected 6 hrs before eyes were enucleated ( $\mathrm{n}=3$ eyes). (B) The corneal epithelium of P60 mice were denuded and then treated with $20 \mu \mathrm{M}$ verteporfin or Rho-Act $(1 \mu \mathrm{g} / \mathrm{ml})$ or vehicle and images were taken at indicated time points ( $n=4$ eyes). (C) Schematic representation of the three transgenes. (D) Potential outcome of Confetti reporter rearrangement and expression in a specific cellular compartment.(E) Schematic representation of the treatment verteporfin or Rho-Act. (F) 2-month-old triple-transgenic (K15GFP; K14Cre ${ }^{\text {ERT; }}$ R26R-Confetti) mice were induced to express Confetti reporters, and limbal epithelium was removed. After limbal removal cells were treated with Vehicle or $20 \mu \mathrm{M}$ verteporfin or Rho-Act $(1 \mu \mathrm{g} / \mathrm{ml})$. For accurate stripe tracking over time, eyes with $1-2 \mathrm{RFP}^{+}$stripes were followed $(\mathrm{n}=2$ eyes). $\mathrm{RFP}^{+}$corneal-committed cells repaired the limbus by day 1 and re-expressed K15-GFP by day 10 in the vehicle, whereas in verteporfin or Rho-Act treated cornea K15-GFP expression was negligible. Scale bars are 50 $\mu \mathrm{m}$. Peri.- Peripheral cornea, LER- Limbal Epithelium Removal, D-Day.

Figure 4. Inhibition of YAP represses LSC phenotype and function. (A, B) Primary hLSCs and epidermal SCs were co-cultured with NIH-3T3 feeder cells and grown on a plastic plate for 4days and the expression of stemness marker (K15, P63), total YAP and pYAP were tested by immunostaining. The golden arrow indicates stem cells and the white arrow indicates differentiated cell regions. (C-F) Primary hLSCs were cultured and maintained at low calcium (Day 0) or induced to differentiate (Day 7) and the expression of the stemness and differentiation $(\mathrm{K} 3, \mathrm{~K} 12)$ marker was tested by quantitative real time PCR (qPCR) (C) or Western blot (D) or immunostaining (E) and quantified for YAP expression(F) (n=25). (GK) Primary hLSCs were transfected with esiRNA against YAP (siYAP) or control esiRNA (siCtl) and 4872 hours later, the expression of YAP was examined by real time PCR $(\mathrm{G})$ and Western lot $(\mathrm{H})$. Quantitative 
real-time PCR analysis of the indicated markers of stem/progenitor cells or markers of differentiated cells (I) was performed, or lysates of cells were subjected to Western blot analysis (H). LSCs were treated with siCtl or siYAP and subjected to clonogenicity test $(\mathrm{K})$ and the number of the colony was quantified (K). (L) Immunohistochemistry of YAP and K15 was performed on paraffin sections human limbus, cornea and skin. The mean nuclear intensity of YAP is shown by Tukey box-and-whisker plot followed by t-test with Welch's correction. Real-time data was normalized to the housekeeping gene (mean \pm standard error of mean, $\mathrm{n}=4$ ) as fold increase compared to control sample statistical significance was assessed by t-test $\left(^{*}, p<\right.$ $.05 ; * *, p<.01 ; * * *, p<.001, * * * *, p<.0001)$. Nuclei were detected by DAPI counterstaining. Scale bars are $50 \mu \mathrm{m}$.

Figure 5. Stiffness-induced actomyosin contractility enhances LATS1/2 phosphorylation and sequesters YAP in the cytoplasm to induce cell differentiation. (A-D) Primary hLSCs were grown on soft or stiff PDMS substrate coated with fibronectin and cultured for 4 days. Expression of stemness marker and differentiation marker was tested by real time PCR (qPCR) (A) or western blot (B). Immunostaining for YAP, pLATS1/2 and vinculin was performed (C) and mean nuclear YAP intensity was quantified (D) $(n=30)$. (E-I) Primary hLSCs were grown on stiff PDMS substrate coated with fibronectin and cultured for 4 days with vehicle/blebbistatin, or cells were grown on soft PDMS substrate coated with fibronectin and cultured for 4 days with vehicle/Rho-Act. Expression of stemness marker, differentiation marker or YAP and pLATS $1 / 2$ was tested by immunostaining $(\mathrm{E}, \mathrm{F})$ or $\mathrm{qPCR}(\mathrm{H}, \mathrm{I})$ and mean nuclear intensity of YAP was quantified $(\mathrm{G})(\mathrm{n}=25)$. (J) Primary hLSCs after overnight incubation on soft (left) and stiff (right) pillars stained for K15 and F-actin (phalloidin). (K) Forces generated by K15-positive and K15-negative cells (undifferentiated and differentiated; see Methods); $\mathrm{n}=44$ and 52 respectively, from $>4$ cells in each case. Mean nuclear intensity of YAP is shown by Tukey box-and-whisker plot followed by t-test for treatment with Bleb and t-test with Welch's correction for treatment with Rho-Act. Force generated by LSCs is shown by Tukey box-and-whisker plot followed by Mann Whitney test. Real-time data were normalized to the housekeeping gene and is presented (mean \pm standard error of mean, $\mathrm{n}=4$ ) as fold increase compared to control sample and statistical analysis was performed by t-test $\left(^{*}, p<.05 ; * *, p<.01 ; * * *, p<.001\right.$, $* * * *, p<.0001)$. Nuclei were detected by DAPI counterstaining. Scale bars are 50 $\mu \mathrm{m}$.

Figure 6. Stiffness-induced differentiation is mediated by SMAD2/3. (A) Primary hLSCs were grown on soft or stiff PDMS substrate coated with fibronectin and cultured for 4 days with vehicle or RhoActivator and immunostained for SMAD2/3. Mean nuclear SMAD2/3 intensity was quantified (B) (n= 980, 316 and 296 respectively). (C-F) Primary hLSCs were grown on soft PDMS substrate coated with fibronectin and treated with TGF $\beta$ ligand for 2 days and immunostained and quantified for SMAD2/3 (C, $\mathrm{D}, \mathrm{n}=239$ and 57 respectively) or differentiation marker K12 (E, F, n=8 and 9 respectively). (G-N) Primary hLSCs were grown on stiff PDMS substrate coated with fibronectin and treated with SB431542 for 2 days and immunostained and quantified for $\operatorname{SMAD} 2 / 3(\mathrm{G}, \mathrm{H}, \mathrm{n}=133$ and 338 respectively), differentiation marker K12 (I, J, n=10 and 9 respectively), pLATS1/2 (K, L, n= 22 and 18 respectively) and YAP (M, $\mathrm{N}, \mathrm{n}=50$ and 72 respectively). Primary hLSCs were grown on soft PDMS substrate coated with fibronectin and treated with verteporfin for 4 days and immunostained for YAP and quantified $(\mathrm{O}, \mathrm{P}, \mathrm{n}=44$ and 50 respectively). Immunohistochemistry of SMAD2/3 was performed on paraffin sections of the P60 mouse cornea. The regions of the limbus and corneal periphery are shown. Mean nuclear SMAD2/3 intensity is shown by the Tukey box-and-whisker plot followed by Mann Whitney test. \% of K12 positive cells is shown by Tukey box-and-whisker plot followed by t-test with Welch's correction $\left(*, p<.05 ;{ }^{* *}, p<.01\right.$; $* * *, p<.001, * * * *, p<.0001)$. Nuclei were detected by DAPI counterstaining. Scale bars are $50 \mu \mathrm{m}$. 
Figure 7. Inhibition of mechano-sensing or TGFß pathway attenuates LSC differentiation on plastic. (A-I) Primary hLSCs were grown on plastic and treated with vehicle or Blebbistatin or SB43152 for 4 days and the expression of stemness and differentiation marker were tested by immunostaining (A, E) or q PCR analysis $(B, F)$ or cells were taken for clonogenicity assay $(C, G)$ and the number of the colony were quantified $(\mathrm{D}, \mathrm{H})$. Real-time data was normalized to the housekeeping gene and is presented (mean \pm standard error of mean, $\mathrm{n}=4$ ) as fold increase compared to control sample and statistical analysis was performed by t-test $\left(*, p<.05 ;{ }^{* *}, p<.01 ; * * *, p<.001, * * * *, p<.0001\right)$. Nuclei were detected by DAPI counterstaining. Scale bars are $50 \mu \mathrm{m}$.

Figure S1. Immunohistochemistry of pLATS1/2 and pYAP in the maturing cornea. (A) Immunohistochemistry of pLATS1/2 and pYAP in P15 and P60 murine cornea. Golden arrowheads indicate the absence of these markers in the limbus region.

Figure S2. Immunohistochemistry of YAP in $\mathrm{P60} \mathrm{Lox}{ }^{\mathrm{OE}}$ murine cornea. (A) Immunohistochemistry of YAP protein was performed on paraffin sections of the P60 WT and Lox ${ }^{\mathrm{OE}}$ mouse cornea. Golden arrowheads indicate the absence of nYAP in the limbus region.

Figure S3. Quantification of proliferation and epithelial healing in the presence of YAP inhibitor or Rho Activator II. (A) Quantification of EdU labeling in different outer, inner limbal zones and corneal periphery (n=3). (B) Quantification of wound area following treatment with YAP inhibitor or Rho Activator II $(\mathrm{n}=4)$. Statistics were tested by two-way ANOVA followed by Tukey's multiple comparisons test $\left(^{*}, p<\right.$ $.05 ; * *, p<.01 ; * * *, p<.001 ; * * * *, p<.0001)$. Data presented as mean \pm standard error of mean.

Figure S4. YAP expression during dedifferentiation. (A) Immunohistochemistry of YAP on limbal and periphery sections of the cornea on indicated days following limbal epithelium removal.

Figure S5. Verteporfin inhibits substrate stiffness-induced LSC differentiation. (A) Primary hLSCs were grown on soft or stiff PDMS substrate coated with fibronectin and grown for 4 days. Expression of stemness marker (P63, K15) and differentiation marker (K12, K3) was tested by immunofluorescence staining or brightfield images were taken (B). (C-E) Primary hLSCs were grown on plastic and grown for 4 days with verteporfin and then taken for by immunofluorescence staining for stemness marker (C) or taken for clonogencity test (D) and the number of the colony was quantified (E). Data presented as mean \pm standard error of mean, $\mathrm{n}=2$ and statistical analysis was performed by $\mathrm{t}$-test $(*, \mathrm{p}<0.05)$. Nuclei were detected by DAPI counterstaining. Scale bars are $50 \mu \mathrm{m}$.

Figure S6. Analysis of LSCs on pillars. (A) Areas of cells as measured using the phalloidin signal after overnight incubation on soft and stiff pillars ( $\mathrm{n}=44$ and 47 respectively). (B) K15 immunostaining fluorescence signal as a function of cell area in cells plated overnight on the soft pillars. The cell area is shown by the Tukey box-and-whisker plot followed by t-test $(*, p<.05 ; * *, p<.01$; ***, $p<.001)$.

Figure S7. Nuclear SMAD2/3 is associated with differentiation. (A) Primary hLSCs were cultured and maintained at low calcium (Day 0) or induced to differentiate (Day7) and immunostained for SMAD2/3 (A) and mean nuclear SMAD2/3 intensity was quantified ( $\mathrm{n}=31$ and 25 respectively) (B). Mean nuclear SMAD2/3 intensity is shown by the Tukey box-and-whisker plot followed by t-test with Welch's correction $(*, p<.05 ; * *, p<.01 ; * * *, p<.001, * * * *, p<.0001)$. Nuclei were detected by DAPI counterstaining. Scale bars is $50 \mu \mathrm{m}$. 


\section{References:}

Altshuler A, Amitai-Lange A, Tarazi N, Dey S, Strinkovsky L, Hadad-Porat S, Bhattacharya S, Nasser W, Imeri J, Ben-David G, et al (2021) Discrete limbal epithelial stem cell populations mediate corneal homeostasis and wound healing. Cell Stem Cell

Amelio I, Lena AM, Viticchie G, Shalom-Feuerstein R, Terrinoni A, Dinsdale D, Russo G, Fortunato C, Bonanno E, Spagnoli LG, et al (2012) miR-24 triggers epidermal differentiation by controlling actin adhesion and cell migration. J Cell Biol 199: 347-363

Amitai-Lange A, Altshuler A, Bubley J, Dbayat N, Tiosano B \& Shalom-Feuerstein R (2015a) Lineage tracing of stem and progenitor cells of the murine corneal epithelium. Stem Cells 33: 230-239

Amitai-Lange A, Berkowitz E, Altshuler A, Dbayat N, Nasser W, Suss-Toby E, Tiosano B \& ShalomFeuerstein R (2015b) A Method for Lineage Tracing of Corneal Cells Using Multi-color Fluorescent Reporter Mice. JoVE: e53370

Baker A-M, Bird D, Lang G, Cox TR \& Erler JT (2013) Lysyl oxidase enzymatic function increases stiffness to drive colorectal cancer progression through FAK. Oncogene 32: 1863-1868

Barker N, van Es JH, Kuipers J, Kujala P, van den Born M, Cozijnsen M, Haegebarth A, Korving J, Begthel H, Peters PJ, et al (2007) Identification of stem cells in small intestine and colon by marker gene Lgr5. Nature 449: 1003-1007

Basu S, Totty NF, Irwin MS, Sudol M \& Downward J (2003) Akt Phosphorylates the Yes-Associated Protein, YAP, to Induce Interaction with 14-3-3 and Attenuation of p73-Mediated Apoptosis. Mol Cell 11: 11-23

Batlle E \& Wilkinson DG (2012) Molecular mechanisms of cell segregation and boundary formation in development and tumorigenesis. Cold Spring Harb Perspect Biol 4: a008227

Bhattacharya S, Serror L, Nir E, Dhiraj D, Altshuler A, Khreish M, Tiosano B, Hasson P, Panman L, Luxenburg C, et al (2019) SOX2 Regulates P63 and Stem/Progenitor Cell State in the Corneal Epithelium. Stem Cells 37: 417-429

Blanpain C \& Fuchs E (2014) Stem cell plasticity. Plasticity of epithelial stem cells in tissue regeneration. Science (80- ) 344: 1242281

Bora-Singhal N, Nguyen J, Schaal C, Perumal D, Singh S, Coppola D \& Chellappan S (2015) YAP1 Regulates OCT4 Activity and SOX2 Expression to Facilitate Self-Renewal and Vascular Mimicry of Stem-Like Cells. Stem Cells 33: 1705-1718 
Bornschlögl T, Bildstein L, Thibaut S, Santoprete R, Fiat F, Luengo GS, Doucet J, Bernard BA \& Baghdadli N (2016) Keratin network modifications lead to the mechanical stiffening of the hair follicle fiber. Proc Natl Acad Sci U S A 113: 5940-5945

Brandão AS, Bensimon-Brito A, Lourenço R, Borbinha J, Soares AR, Mateus R \& Jacinto A (2019) Yap induces osteoblast differentiation by modulating Bmp signalling during zebrafish caudal fin regeneration. J Cell Sci 132: jcs231993

Burridge K, Monaghan-Benson E \& Graham DM (2019) Mechanotransduction: from the cell surface to the nucleus via RhoA. Philos Trans R Soc B Biol Sci 374: 20180229

Camargo FD, Gokhale S, Johnnidis JB, Fu D, Bell GW, Jaenisch R \& Brummelkamp TR (2007) YAP1 increases organ size and expands undifferentiated progenitor cells. Curr Biol 17: 2054-2060

Cohen-Kaplan V, Naroditsky I, Zetser A, Ilan N, Vlodavsky I \& Doweck I (2008) Heparanase induces VEGF C and facilitates tumor lymphangiogenesis. Int J Cancer 123: 2566-2573

Collinson JM, Morris L, Reid AI, Ramaesh T, Keighren MA, Flockhart JH, Hill RE, Tan S-S, Ramaesh $\mathrm{K}$, Dhillon B, et al (2002) Clonal analysis of patterns of growth, stem cell activity, and cell movement during the development and maintenance of the murine corneal epithelium. Dev Dyn 224: $432-440$

Cotsarelis G, Sun TT \& Lavker RM (1990) Label-retaining cells reside in the bulge area of pilosebaceous unit: Implications for follicular stem cells, hair cycle, and skin carcinogenesis. Cell 61: 1329-1337

Dahmann C, Oates AC \& Brand M (2011) Boundary formation and maintenance in tissue development. Nat Rev Genet 12: 43-55

Dorà NJ, Hill RE, Collinson JM \& West JD (2015) Lineage tracing in the adult mouse corneal epithelium supports the limbal epithelial stem cell hypothesis with intermittent periods of stem cell quiescence. Stem Cell Res 15: 665-677

Dupont S, Morsut L, Aragona M, Enzo E, Giulitti S, Cordenonsi M, Zanconato F, Le Digabel J, Forcato M, Bicciato S, et al (2011) Role of YAP/TAZ in mechanotransduction. Nature 474: 179

Eberwein P, Nohava J, Schlunck G \& Swain M (2014) Nanoindentation Derived Mechanical Properties of the Corneoscleral Rim of the Human Eye. Key Eng Mater 606: 117-120

Edelstein A, Amodaj N, Hoover K, Vale R \& Stuurman N (2010) Computer control of microscopes using $\mu$ Manager. Curr Protoc Mol Biol Chapter 14: Unit14.20

Elosegui-Artola A, Oria R, Chen Y, Kosmalska A, Pérez-González C, Castro N, Zhu C, Trepat X \& 
Roca-Cusachs P (2016) Mechanical regulation of a molecular clutch defines force transmission and transduction in response to matrix rigidity. Nat Cell Biol 18: 540-548

van Es JH, Sato T, van de Wetering M, Lyubimova A, Yee Nee AN, Gregorieff A, Sasaki N, Zeinstra L, van den Born M, Korving J, et al (2012) Dll1+ secretory progenitor cells revert to stem cells upon crypt damage. Nat Cell Biol 14: 1099-1104

Fatima A, Iftekhar G, Sangwan VS \& Vemuganti GK (2007) Ocular surface changes in limbal stem cell deficiency caused by chemical injury: a histologic study of excised pannus from recipients of cultured corneal epithelium. Eye 22: 1161

Feld L, Kellerman L, Mukherjee A, Livne A, Bouchbinder E \& Wolfenson H (2020) Cellular contractile forces are nonmechanosensitive. Sci Adv 6: eaaz6997

Fuchs E, Tumbar T \& Guasch G (2004) Socializing with the Neighbors : Stem Cells and Their Niche. Cell 116: 769-778

Gabay Yehezkely R, Zaffryar-Eilot S, Kaganovsky A, Fainshtain Malka N, Aviram R, Livneh I \& Hasson P (2020) Intracellular Role for the Matrix-Modifying Enzyme Lox in Regulating Transcription Factor Subcellular Localization and Activity in Muscle Regeneration. Dev Cell 53: 406-417.e5

Ghareeb AE, Lako M \& Figueiredo FC (2020) Recent Advances in Stem Cell Therapy for Limbal Stem Cell Deficiency: A Narrative Review. Ophthalmol Ther 9: 809-831

Ghassemi S, Meacci G, Liu S, Gondarenko AA, Mathur A, Roca-Cusachs P, Sheetz MP \& Hone J (2012) Cells test substrate rigidity by local contractions on submicrometer pillars. Proc Natl Acad Sci U S A 109: 5328-5333

Giacomini MM, Travis MA, Kudo M \& Sheppard D (2012) Epithelial cells utilize cortical actin/myosin to activate latent TGF- $\beta$ through integrin $\alpha(\mathrm{v}) \beta(6)$-dependent physical force. Exp Cell Res 318: 716722

Di Girolamo N, Bobba S, Raviraj V, Delic NC, Slapetova I, Nicovich PR, Halliday GM, Wakefield D, Whan R \& Lyons JG (2015) Tracing the Fate of Limbal Epithelial Progenitor Cells in the Murine Cornea. Stem Cells 33: 157-169

Gouveia RM, Lepert G, Gupta S, Mohan RR, Paterson C \& Connon CJ (2019) Assessment of corneal substrate biomechanics and its effect on epithelial stem cell maintenance and differentiation. Nat Commun 10: 1496

Halder G, Dupont S \& Piccolo S (2012) Transduction of mechanical and cytoskeletal cues by YAP and TAZ. Nat Rev Mol Cell Biol 13: 591-600 
Han D, Byun S-H, Park S, Kim J, Kim I, Ha S, Kwon M \& Yoon K (2015) YAP/TAZ enhance mammalian embryonic neural stem cell characteristics in a Tead-dependent manner. Biochem Biophys Res Commun 458: 110-116

Han K-Y, Tran JA, Chang J-H, Azar DT \& Zieske JD (2017) Potential role of corneal epithelial cellderived exosomes in corneal wound healing and neovascularization. Sci Rep 7: 40548

Judson RN, Tremblay AM, Knopp P, White RB, Urcia R, De Bari C, Zammit PS, Camargo FD \& Wackerhage H (2012) The Hippo pathway member Yap plays a key role in influencing fate decisions in muscle satellite cells. J Cell Sci 125: 6009-6019

Kasetti RB, Gaddipati S, Tian S, Xue L, Kao WW-Y, Lu Q \& Li Q (2016) Study of corneal epithelial progenitor origin and the Yap1 requirement using keratin 12 lineage tracing transgenic mice. Sci Rep 6: 35202

Kim S, Thomasy SM, Raghunathan VK, Teixeira LBC, Moshiri A, FitzGerald P \& Murphy CJ (2019) Ocular phenotypic consequences of a single copy deletion of the Yap1 gene (Yap1 (+/-)) in mice. Mol Vis 25: 129-142

Komuro A, Nagai M, Navin NE \& Sudol M (2003) WW Domain-containing Protein YAP Associates with ErbB-4 and Acts as a Co-transcriptional Activator for the Carboxyl-terminal Fragment of ErbB-4 That Translocates to the Nucleus *. 278: 33334-33341

Lena a M, Shalom-Feuerstein R, Rivetti di Val Cervo P, Aberdam D, Knight R a, Melino G \& Candi E (2008) miR-203 represses ‘stemness' by repressing DeltaNp63. Cell Death Differ 15: 1187-1195

Lessey EC, Guilluy C \& Burridge K (2012) From Mechanical Force to RhoA Activation. Biochemistry 51: $7420-7432$

Levental KR, Yu H, Kass L, Lakins JN, Egeblad M, Erler JT, Fong SFT, Csiszar K, Giaccia A, Weninger W, et al (2009) Matrix Crosslinking Forces Tumor Progression by Enhancing Integrin Signaling. Cell 139: 891-906

Lin B, Srikanth P, Castle AC, Nigwekar S, Malhotra R, Galloway JL, Sykes DB \& Rajagopal J (2018a) Modulating Cell Fate as a Therapeutic Strategy. Cell Stem Cell 23: 329-341

Lin B, Srikanth P, Castle AC, Nigwekar S, Malhotra R, Galloway JL, Sykes DB \& Rajagopal J (2018b) Modulating Cell Fate as a Therapeutic Strategy. Cell Stem Cell 23: 329-341

Lorthongpanich C, Thumanu K, Tangkiettrakul K, Jiamvoraphong N, Laowtammathron C, Damkham N, U-pratya Y \& Issaragrisil S (2019) YAP as a key regulator of adipo-osteogenic differentiation in human MSCs. Stem Cell Res Ther 10: 402 
Ma S, Meng Z, Chen R \& Guan K-L (2019) The Hippo Pathway: Biology and Pathophysiology. Annu Rev Biochem 88: 577-604

Maruri DP, Miron-Mendoza M, Kivanany PB, Hack JM, Schmidtke DW, Petroll WM \& Varner VD (2020) ECM Stiffness Controls the Activation and Contractility of Corneal Keratocytes in Response to TGF- $\beta 1$. Biophys J 119: 1865-1877

Massagué J (1998) TGF-beta signal transduction. Annu Rev Biochem 67: 753-791

Meacci G, Wolfenson H, Liu S, Stachowiak MR, Iskratsch T, Mathur A, Ghassemi S, Gauthier N, Tabdanov E, Lohner J, et al (2016) $\alpha$-Actinin links extracellular matrix rigidity-sensing contractile units with periodic cell-edge retractions. Mol Biol Cell 27: 3471-3479

Monge C, DiStasio N, Rossi T, Sébastien M, Sakai H, Kalman B, Boudou T, Tajbakhsh S, Marty I, Bigot A, et al (2017) Quiescence of human muscle stem cells is favored by culture on natural biopolymeric films. Stem Cell Res Ther 8: 104

Morris RJ, Liu Y, Marles L, Yang Z, Trempus C, Li S, Lin JS, Sawicki JA \& Cotsarelis G (2004)

Capturing and profiling adult hair follicle stem cells. Nat Biotechnol 22: 411-417

Mou H, Vinarsky V, Tata PR, Brazauskas K, Choi SH, Crooke AK, Zhang B, Solomon GM, Turner B, Bihler H, et al (2016) Dual SMAD Signaling Inhibition Enables Long-Term Expansion of Diverse Epithelial Basal Cells. Cell Stem Cell 19: 217-231

Nagosa S, Leesch F, Putin D, Bhattacharya S, Altshuler A, Serror L, Amitai-Lange A, Nasser W, Aberdam E, Rouleau M, et al (2017) microRNA-184 Induces a Commitment Switch to Epidermal Differentiation. Stem Cell Reports 9: 1991-2004

Nasser W, Amitai-Lange A, Soteriou D, Hanna R, Tiosano B, Fuchs Y \& Shalom-Feuerstein R (2018a) Corneal-Committed Cells Restore the Stem Cell Pool and Tissue Boundary following Injury. Cell Rep 22: 323-331

Nasser W, Amitai-Lange A, Soteriou D, Hanna R, Tiosano B, Fuchs Y \& Shalom-Feuerstein R (2018b) Corneal-Committed Cells Restore the Stem Cell Pool and Tissue Boundary following Injury. Cell Rep 22: 323-331

Nasser W, Amitai-Lange A, Soteriou D, Hanna R, Tiosano B, Fuchs Y \& Shalom-Feuerstein R (2018c) Corneal-Committed Cells Restore the Stem Cell Pool and Tissue Boundary following Injury. Cell Rep 22: 323-331

O'Callaghan AR \& Daniels JT (2011) Concise review: limbal epithelial stem cell therapy: controversies and challenges. Stem Cells 29: 1923-1932 
Raghunathan VK, Dreier B, Morgan JT, Tuyen BC, Rose BW, Reilly CM, Russell P \& Murphy CJ (2014) Involvement of YAP, TAZ and HSP90 in contact guidance and intercellular junction formation in corneal epithelial cells. PLoS One 9: e109811-e109811

Richardson A, Lobo EP, Delic NC, Myerscough MR, Lyons JG, Wakefield D \& Di Girolamo N (2017) Keratin-14-Positive Precursor Cells Spawn a Population of Migratory Corneal Epithelia that Maintain Tissue Mass throughout Life. Stem Cell Reports 9: 1081-1096

Rompolas P, Mesa KR \& Greco V (2013) Spatial organization within a niche as a determinant of stemcell fate. Nature 502: 513-518

Sampath Narayanan A, Siegel RC \& Martin GR (1972) On the inhibition of lysyl oxidase by $\beta$ aminopropionitrile. Biochem Biophys Res Commun 46: 745-751

Schlegelmilch K, Mohseni M, Kirak O, Pruszak J, Rodriguez JR, Zhou D, Kreger BT, Vasioukhin V, Avruch J, Brummelkamp TR, et al (2011) Yap1 acts downstream of $\alpha$-catenin to control epidermal proliferation. Cell 144: 782-795

Shalom-Feuerstein R, Lena AM, Zhou H, De La Forest Divonne S, Van Bokhoven H, Candi E, Melino G \& Aberdam D (2011) $\triangle \mathrm{Np} 63$ is an ectodermal gatekeeper of epidermal morphogenesis. Cell Death Differ 18: 887-896

Shi M, Zhu J, Wang R, Chen X, Mi L, Walz T \& Springer TA (2011) Latent TGF- $\beta$ structure and activation. Nature 474: 343-349

Spradling A, Drummond-barbosa D \& Kai T (2001) Stem cells find their niche. Nature 414

Stepp MA (2006) Corneal integrins and their functions. Exp Eye Res 83: 3-15

Tetteh PW, Basak O, Farin HF, Wiebrands K, Kretzschmar K, Begthel H, van den Born M, Korving J, de Sauvage F, van Es JH, et al (2016) Replacement of Lost Lgr5-Positive Stem Cells through Plasticity of Their Enterocyte-Lineage Daughters. Cell Stem Cell 18: 203-213

Tomlinson V, Gudmundsdottir K, Luong P, Leung K-Y, Knebel A \& Basu S (2010) JNK phosphorylates Yes-associated protein (YAP) to regulate apoptosis. Cell Death Dis 1: e29-e29

Totaro A, Castellan M, Battilana G, Zanconato F, Azzolin L, Giulitti S, Cordenonsi M \& Piccolo S (2017) YAP/TAZ link cell mechanics to Notch signalling to control epidermal stem cell fate. Nat Commun 8: 15206

Vallet SD \& Ricard-Blum S (2019) Lysyl oxidases: from enzyme activity to extracellular matrix crosslinks. Essays Biochem 63: 349-364 
Vassilev A, Kaneko KJ, Shu H, Zhao Y \& Depamphilis ML (2001) TEAD / TEF transcription factors utilize the activation domain of YAP65, a Src / Yes-associated protein localized in the cytoplasm. 15: 1229-1241

Vattulainen M, Ilmarinen T, Koivusalo L, Viiri K, Hongisto H \& Skottman H (2019) Modulation of Wnt/BMP pathways during corneal differentiation of hPSC maintains ABCG2-positive LSC population that demonstrates increased regenerative potential. Stem Cell Res Ther 10: 236

Walko G, Woodhouse S, Pisco AO, Rognoni E, Liakath-Ali K, Lichtenberger BM, Mishra A, Telerman SB, Viswanathan P, Logtenberg M, et al (2017) A genome-wide screen identifies YAP/WBP2 interplay conferring growth advantage on human epidermal stem cells. Nat Commun 8: 1-16

Wolfenson H, Meacci G, Liu S, Stachowiak MR, Iskratsch T, Ghassemi S, Roca-Cusachs P, O’Shaughnessy B, Hone J \& Sheetz MP (2016) Tropomyosin controls sarcomere-like contractions for rigidity sensing and suppressing growth on soft matrices. Nat Cell Biol 18: 33-42

Yimlamai D, Christodoulou C, Galli GG, Yanger K, Pepe-Mooney B, Gurung B, Shrestha K, Cahan P, Stanger BZ \& Camargo FD (2014) Hippo pathway activity influences liver cell fate. Cell 157: $1324-1338$

Zhang C, Lee HJ, Shrivastava A, Wang R, McQuiston TJ, Challberg SS, Pollok BA \& Wang T (2018) Long-Term In Vitro Expansion of Epithelial Stem Cells Enabled by Pharmacological Inhibition of PAK1-ROCK-Myosin II and TGF- $\beta$ Signaling. Cell Rep 25: 598-610.e5

Zhang H, Pasolli HA \& Fuchs E (2011) Yes-associated protein (YAP) transcriptional coactivator functions in balancing growth and differentiation in skin. Proc Natl Acad Sci U S A 108: 22702275

Zhao B, Li L, Tumaneng K, Wang C-Y \& Guan K-L (2010) A coordinated phosphorylation by Lats and CK1 regulates YAP stability through SCF(beta-TRCP). Genes Dev 24: 72-85

Zhao R, Fallon TR, Saladi SV, Pardo-Saganta A, Villoria J, Mou H, Vinarsky V, Gonzalez-Celeiro M, Nunna N, Hariri LP, et al (2014) Yap Tunes Airway Epithelial Size and Architecture by Regulating the Identity, Maintenance, and Self-Renewal of Stem Cells. Dev Cell 30: 151-165

Zheng Y \& Pan D (2019) The Hippo Signaling Pathway in Development and Disease. Dev Cell 50: 264282 


\section{A}
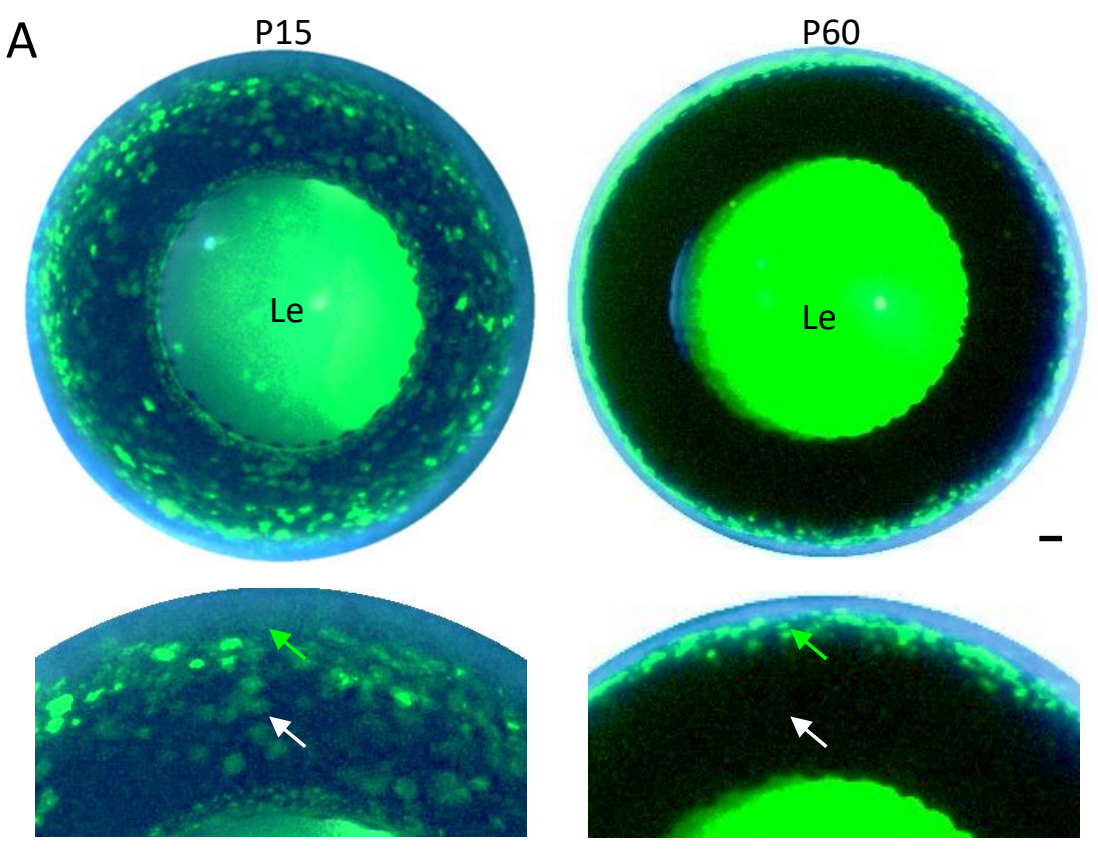

B

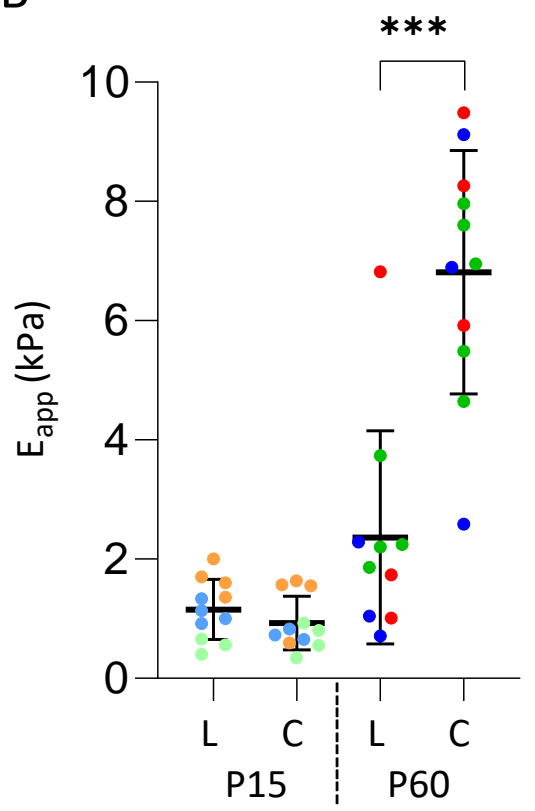

C
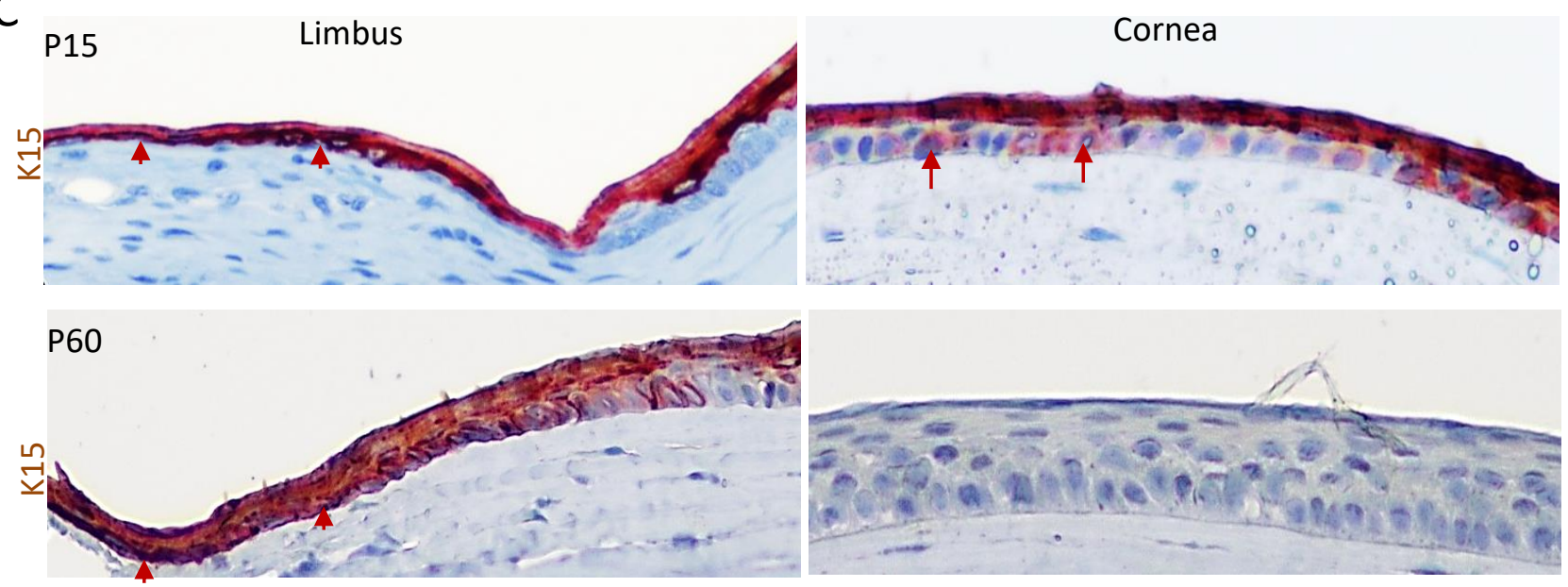

P15
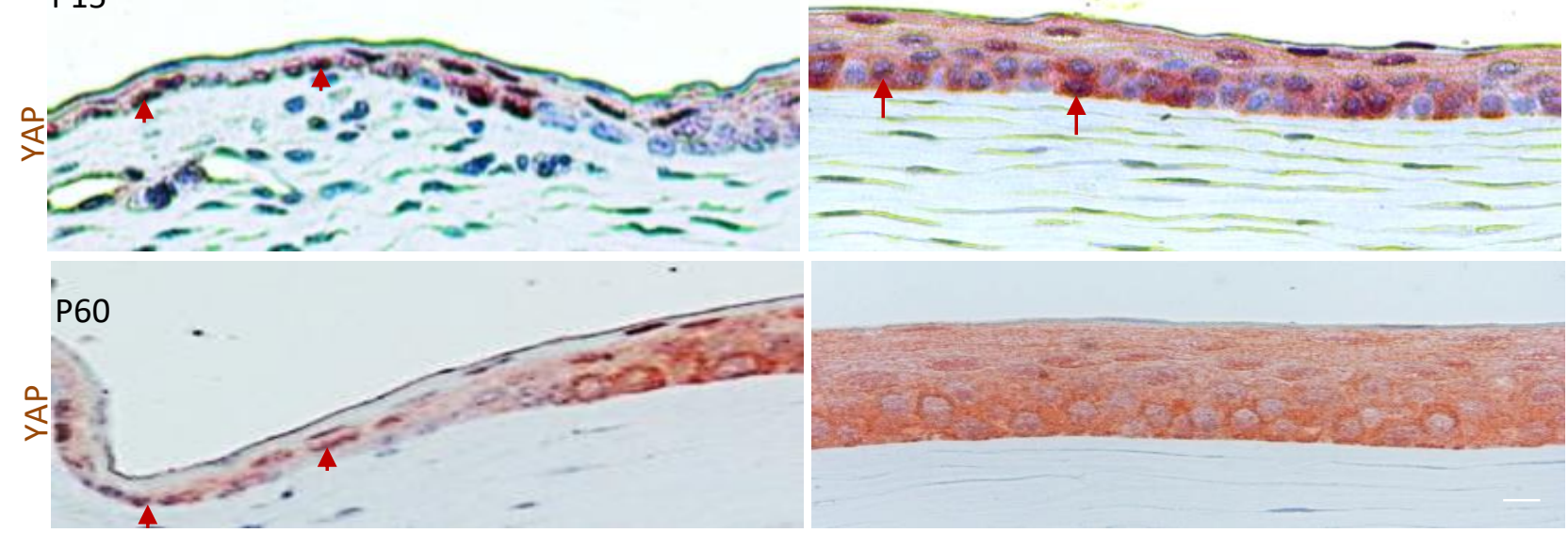

Fig 1. The Development of SC and differentiation compartment is linked with a switch in rigidity and YAP localization 
A
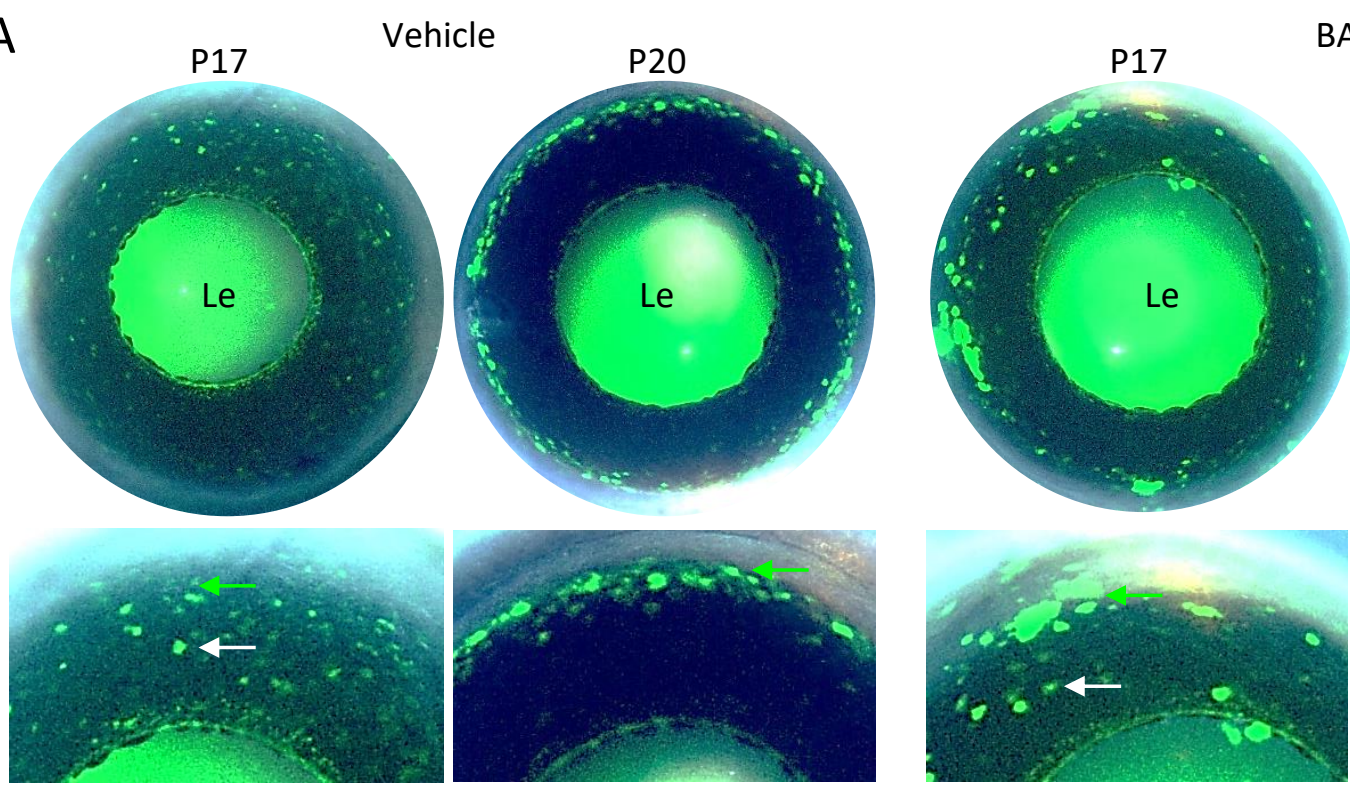

BAPN

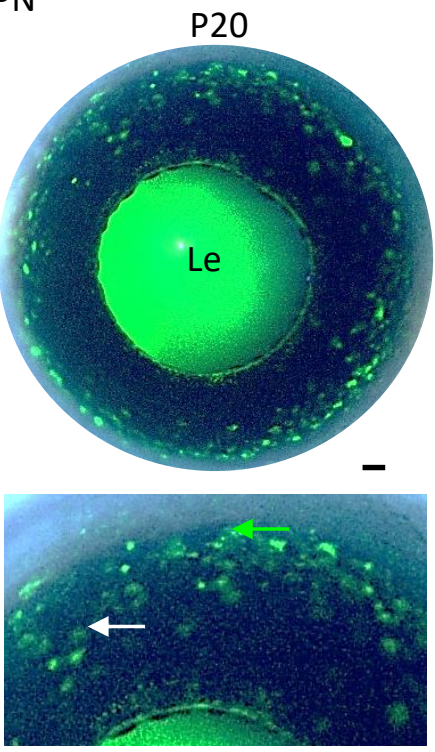

B
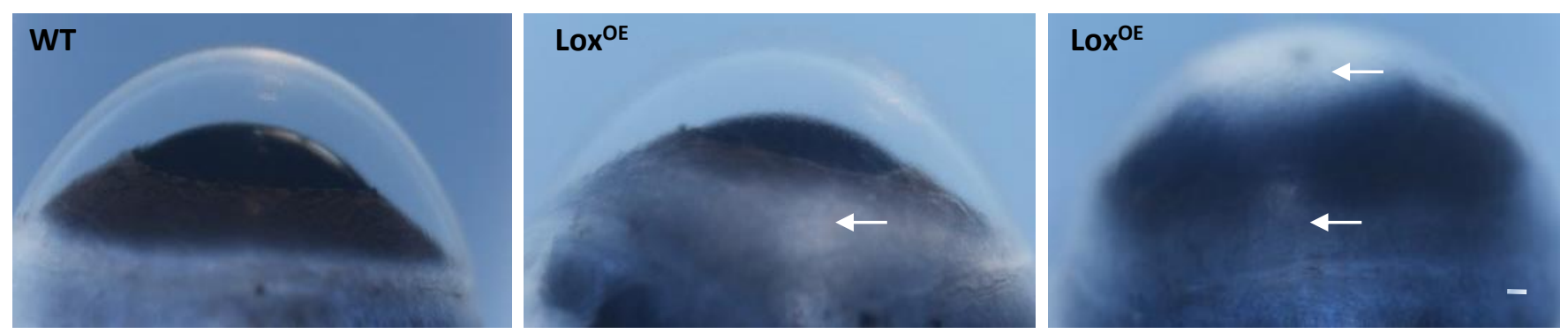

C
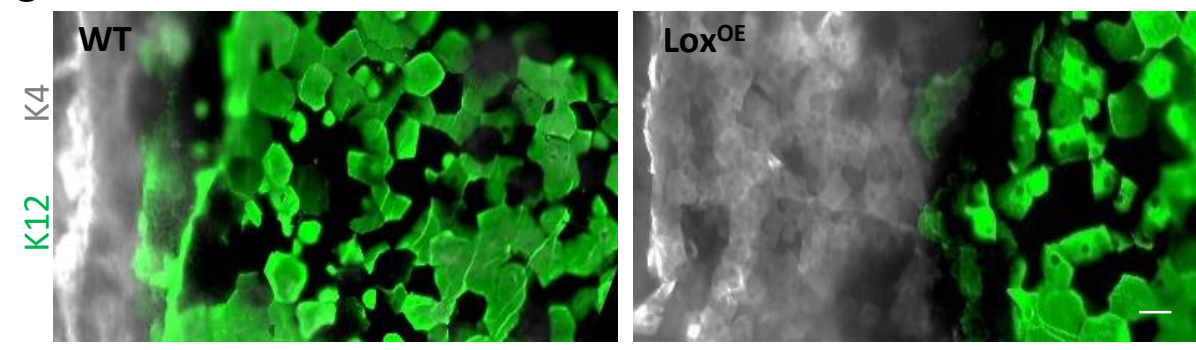

Fig 2. Modulation of Lox crosslinking activity disrupts SC compartmentalization and function 

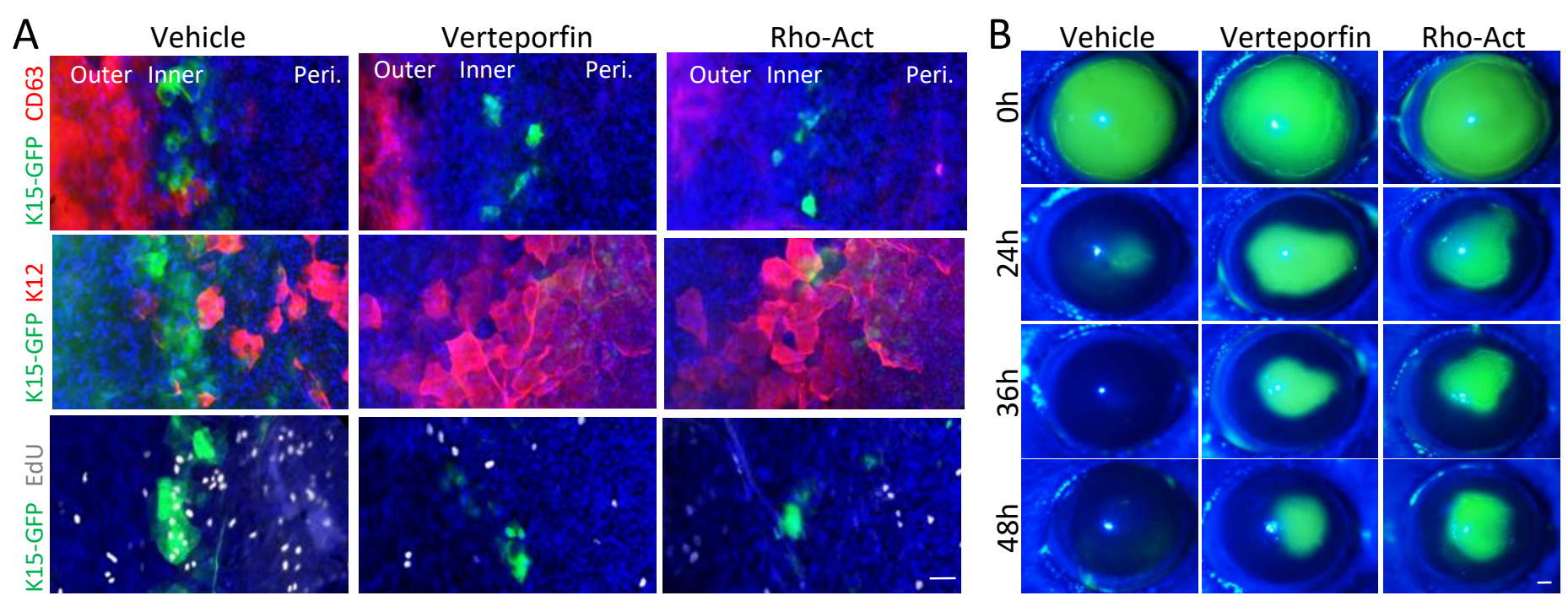

C

$\mathrm{F}$
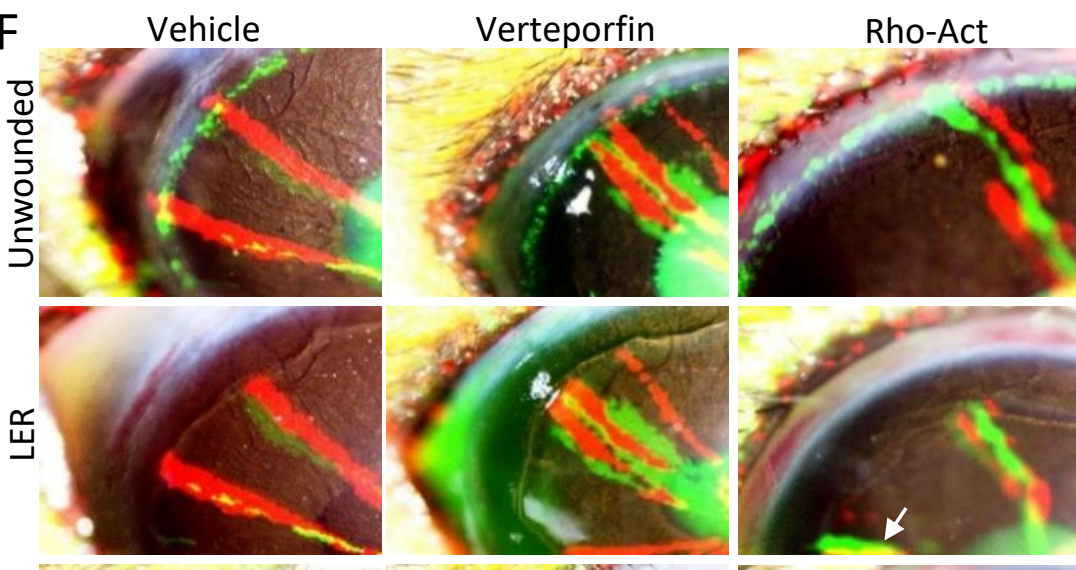

D

Tamoxifen injection

$\mathrm{CAGG} \longrightarrow$ GFP- $d \exists \lambda<\square$

$\mathrm{CAGG} \longrightarrow \mathrm{YFP}-\mathrm{d}=0 \downarrow$

CAGG $\longrightarrow$ RFP-d $\triangle O 4-0$

$\mathrm{CAGG} \longrightarrow \overrightarrow{\mathrm{CFP}} \mathrm{d} \mathrm{d} \mathrm{U}-\mathrm{O}$

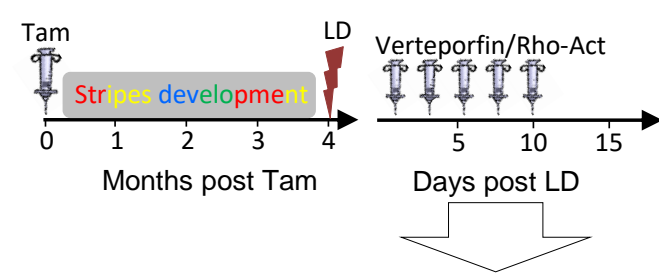

Vital microscopy
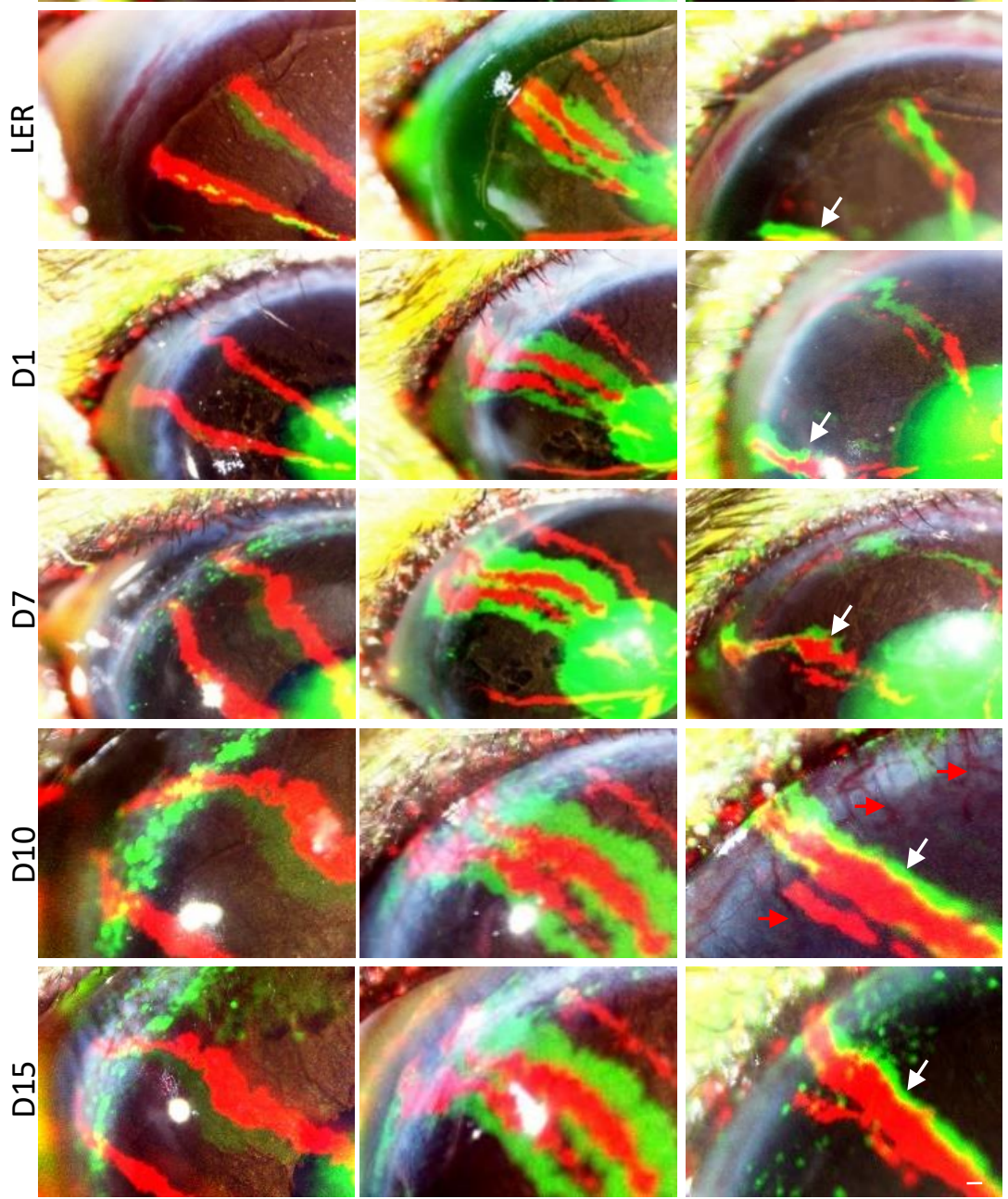

Fig 3. Interference of mechano-transduction pathway through YAP inhibitor or Rho activator impaired LSC function and dedifferentiation 

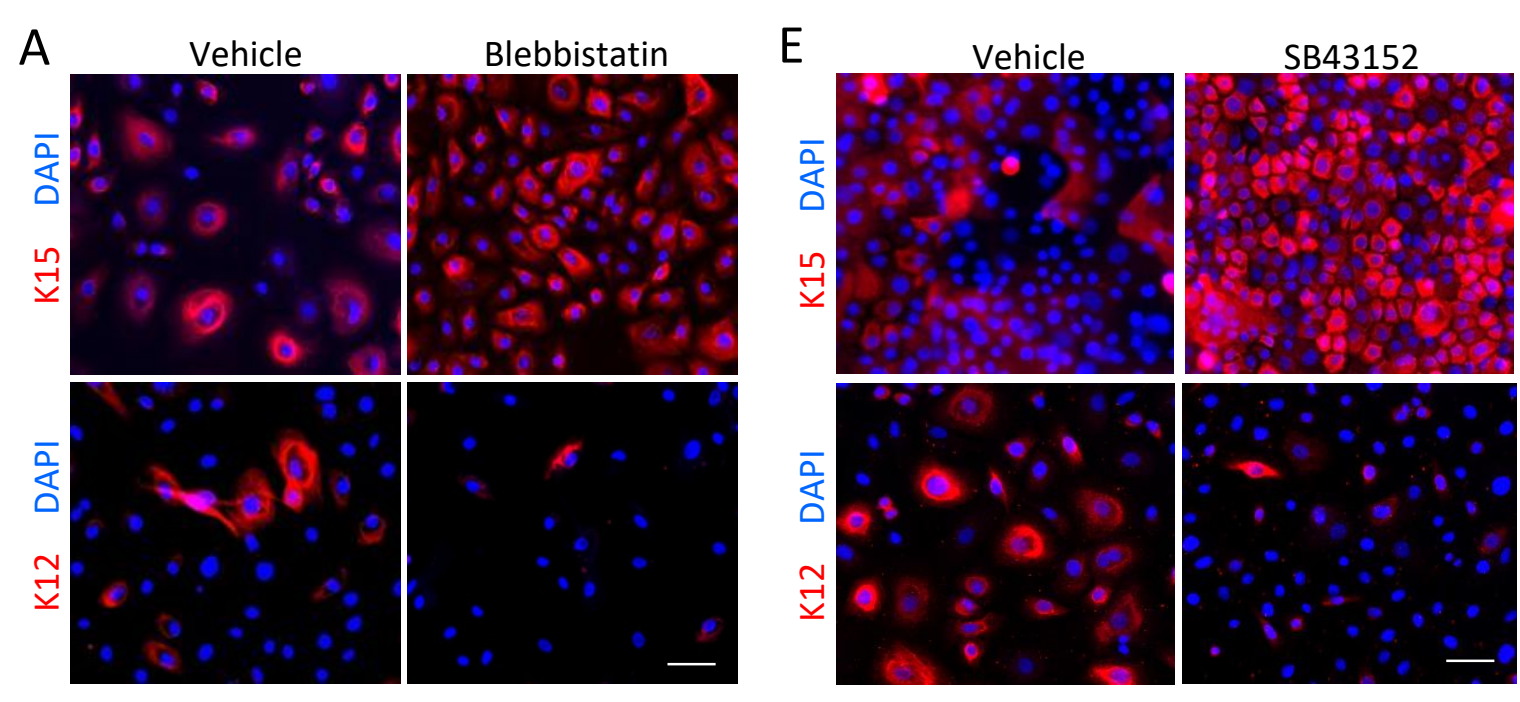

B

Vehicle Blebbistatin

$\mathrm{F}$

Dehicle $\square$ SB431542
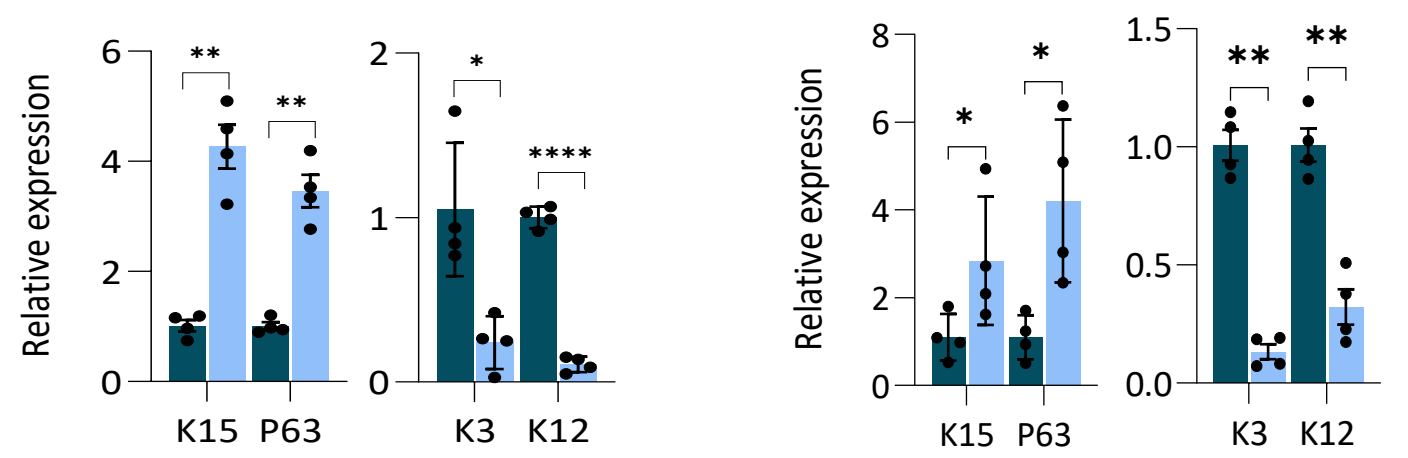

C

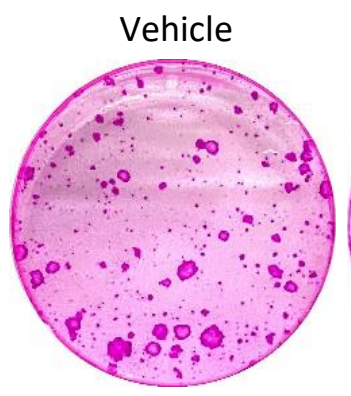

Blebbistatin

G

Vehicle

SB431542
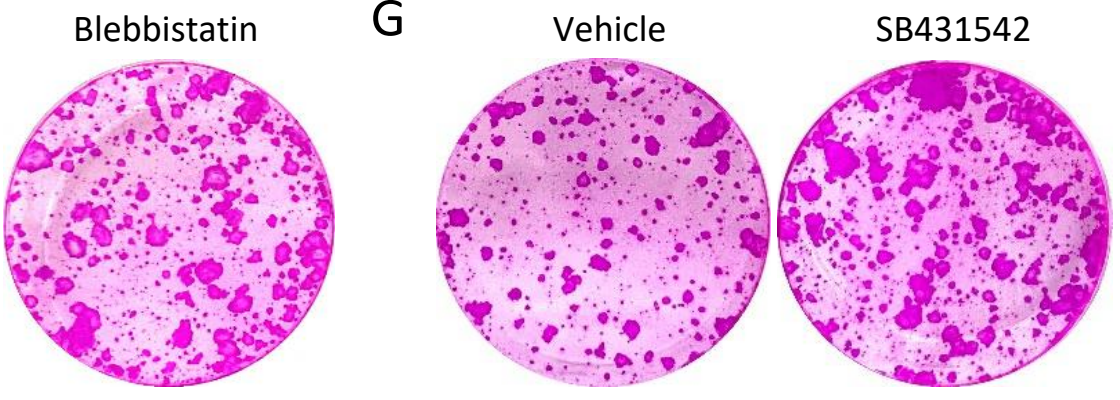

D

$\mathrm{H}$

z
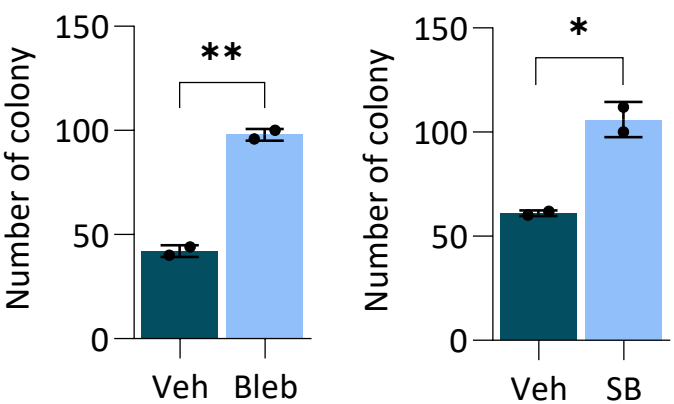

Fig 7: Inhibition of mechano-sensing or TGF $\beta$ pathway attenuates LSC differentiation on plastic 